2019 International Conference on Information and Communications Technology (ICOIACT) took place 24-25 July 2019 in lindo1/172019

Yogyakarta, Indonesia.

$\begin{array}{ll}\text { IEEE catalog number: } & \text { CFP19L86-ART } \\ \text { ISBN: } & 978-1-7281-1655-6\end{array}$

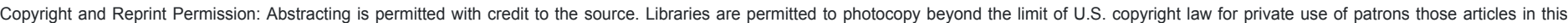

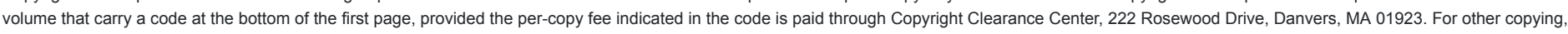
reprint or republication permission, write to IEEE Copyrights Manager, IEEE Operations Center, 445 Hoes Lane, Piscataway, NJ 08854 . All rights reserved. Copyright @ 2019 by IEEE. 


\section{General Chair}

Arief Setyanto (Universitas AMIKOM Yogyakarta, Indonesia)

\section{Publication Chair}

Akhmad Dahlan (Universitas Amikom Yogyakarta, Indonesia)

\section{Secretary}

Nila Puspitasari (Universitas AMIKOM Yogyakarta, Indonesia)

\section{Treasurer}

Sumarni Adi (Universitas AMIKOM Yogyakarta, Indonesia)

\section{Chair of Technical Program Committee}

Kusrini Kusrini (AMIKOM Yogyakarta University, Indonesia)

Emha Taufiq Luthfi (Universitas AMIKOM Yogyakarta, Indonesia)

Ferry Wahyu Wibowo (Universitas Amikom Yogyakarta, Indonesia) 
Technical Program Committee

\begin{tabular}{|c|c|c|}
\hline Intan Ermahani A. Jalil & Universiti Teknikal Malaysia Melaka & Malaysia \\
\hline Mohd Helmy Abd Wahab & Universiti Tun Hussein Onn Malaysia & Malaysia \\
\hline Mohd Hakim Abdul Hamid & Faculty of Information Technology and Communication, UTeM & Malaysia \\
\hline Sumarni Adi & Universitas AMIKOM Yogyakarta & Indonesia \\
\hline David Agustriawan & Indonesia International Institute for Life Sciences (i3L) & Indonesia \\
\hline Mohd Khairul Ikhwan Ahmad & Universiti Tun Hussein Onn Malaysia & Malaysia \\
\hline Anas AISobeh & Yarmouk University & Jordan \\
\hline Dhani Ariatmanto & Universitas Amikom Yogyakarta & Indonesia \\
\hline Ahmad Ashari & Gadjah Mada University & Indonesia \\
\hline Media Ayu & Sampoerna University & Indonesia \\
\hline Azizul Azizan & Universiti Teknologi Malaysia (UTM) & Malaysia \\
\hline Azreen Azman & Universiti Putra Malaysia & Malaysia \\
\hline Aslina Baharum & Universiti Malaysia Sabah & Malaysia \\
\hline Norasiken Bakar & Universiti Teknikal Malaysia Melaka & Malaysia \\
\hline Aashish Bardekar & Sipna College of Engineering and Technology, Amravati & India \\
\hline I Putu Agung Bayupati & Udayana University & Indonesia \\
\hline Indra Budi & Faculty of Computer Science & Indonesia \\
\hline Alessandro Carrega & CNIT & Italy \\
\hline Maria Chiara Caschera & CNR & Italy \\
\hline Mu-Song Chen & Electrical Engineering, Da-Yeh University & Taiwan \\
\hline Wichian Chutimaskul & King Mongkut's University of Technology Thonburi & Thailand \\
\hline Domenico Ciuonzo & University of Naples Federico II, IT & Italy \\
\hline Senthilkumar CP & Auburn University & USA \\
\hline Akhmad Dahlan & Universitas Amikom Yogyakarta & Indonesia \\
\hline Frista Damayanti & Universitas Amikom Yogyakarta & Indonesia \\
\hline Ahmad Fajar & Bina Nusantara University & Indonesia \\
\hline Gianluigi Ferrari & University of Parma & Italy \\
\hline Alireza Ghasempour & ICT Faculty & USA \\
\hline
\end{tabular}




\begin{tabular}{|c|c|c|}
\hline Razvan Andrei Gheorghiu & Politehnica University of Bucharest & Romania \\
\hline Hamdani Hamdani & Universitas Mulawarman & Indonesia \\
\hline Byeong-jun Han & Soongsil University & Korea \\
\hline Seng Hansun & Universitas Multimedia Nusantara & Indonesia \\
\hline K Haribabu & BITS Pilani & India \\
\hline Aslinda Hassan & Universiti Teknikal Malaysia Melaka & Malaysia \\
\hline Roberto Carlos Herrera Lara & National Polytechnic School & Ecuador \\
\hline Tonny Hidayat & Universitas АМІКОМ Yogyakarta & Indonesia \\
\hline Danial Hooshyar & Korea University & Korea \\
\hline Nurulisma Ismail & Universiti Malaysia Perlis & Malaysia \\
\hline Ramkumar Jaganathan & VLB Janakiammal College of Arts and Science & India \\
\hline Arihant Jain & Jaipur Engineering College \& Research Centre & India \\
\hline Muhammad Herman Jamaluddin & Universiti Teknikal Malaysia Melaka & Malaysia \\
\hline Biao Jiang & The City University of New York & USA \\
\hline Sokratis Katsikas & Norwegian University of Science and Technology & Norway \\
\hline Mohammad Khalily Dermany & Islamic Azad University, Khomein Branch & Iran \\
\hline Hasan Ali Khattak & COMSATS University, Islamabad & Pakistan \\
\hline Fukuro Koshiji & Tokyo Polytechnic University & Japan \\
\hline Krisnawati Krisnawati & University of AMIKOM Yogyakarta & Indonesia \\
\hline Rakesh Kumar & National Institute of Technical Teachers Training \& Research & India \\
\hline Kusnawi Kusnawi & AMIKOM University & Indonesia \\
\hline Kusrini Kusrini & AMIKOM Yogyakarta University & Indonesia \\
\hline Armin Lawi & Hasanuddin University & Indonesia \\
\hline Suryadiputra Liawatimena & Bina Nusantara University & Indonesia \\
\hline Linawati Linawati & Universitas Udayana & Indonesia \\
\hline Pavel Loskot & Swansea University & $\begin{array}{l}\text { United Kingdom (Great } \\
\text { Britain) }\end{array}$ \\
\hline Emha Taufiq Luthfi & Universitas AMIKOM Yogyakarta & Indonesia \\
\hline Mahdin Mahboob & Stony Brook University & USA \\
\hline
\end{tabular}




\begin{tabular}{|c|c|c|}
\hline Prita Dewi Mariyam & Universitas Indonesia & Indonesia \\
\hline Ahmed Mobashsher & The University of Queensland & Australia \\
\hline Kamaludin Mohamad Yusof & Universiti Teknologi Malaysia & Malaysia \\
\hline $\begin{array}{l}\text { Seyed Sahand Mohammadi } \\
\text { Ziabari }\end{array}$ & Vrije University of Amsterdam & The Netherlands \\
\hline Mohamed Moharam & Misr University For Science and Technolgy & Egypt \\
\hline Othman Mohd & Universiti Teknikal Malaysia Melaka & Malaysia \\
\hline Ayan Mondal & Indian Institute of Technology, Kharagpur & India \\
\hline Amrit Mukherjee & Jiangsu University & P.R. China \\
\hline Syibrah Naim & Universiti Sains Malaysia & Malaysia \\
\hline N Nasimuddin & Institute for Infocomm Research & Singapore \\
\hline Asro Nasiri & University of Amikom Yogyakarta & Indonesia \\
\hline Shah Nazir & University of Peshawar & Pakistan \\
\hline Ponrudee Netisopakul & King Mongkut's Institute of Technology Ladkrabang & Thailand \\
\hline Md Asri Ngadi & Universiti Teknologi Malaysia & Malaysia \\
\hline Ruzelita Ngadiran & Universiti Malaysia Perlis & Malaysia \\
\hline Hea Choon Ngo & Universiti Teknikal Malaysia Melaka & Malaysia \\
\hline Atsushi Nunome & Kyoto Institute of Technology & Japan \\
\hline Nitish Ojha & DIT University, Dehradun & India \\
\hline Henry Palit & Petra Christian University & Indonesia \\
\hline Jae-Hyun Park & Chung-Ang University & Korea \\
\hline Shahril Parumo & Universiti Teknikal Malaysia Melaka & Indonesia \\
\hline Kiran Sree Pokkuluri & Shri Vishnu Engineering College for Women & India \\
\hline Gede Pramudya Ananta & Universiti Teknikal Malaysia Melaka & Malaysia \\
\hline Nila Puspitasari & Universitas AMIKOM Yogyakarta & Indonesia \\
\hline Lesnanto Multa Putranto & UGM & Indonesia \\
\hline Yuansong Qiao & Athlone Institute of Technology & Ireland \\
\hline Basit Qureshi & University of Bradford & $\begin{array}{l}\text { United Kingdom (Great } \\
\text { Britain) }\end{array}$ \\
\hline
\end{tabular}




\begin{tabular}{|c|c|c|}
\hline Ali Rafiei & University of Technology Sydney & Australia \\
\hline Hemant Kumar Rath & Tata Consultancy Services & India \\
\hline Eric Renault & Institut Mines-Telecom -- Telecom SudParis & France \\
\hline Bagus Rintyarna & Sepuluh Nopember Institute of Technology & Indonesia \\
\hline Sayantam Sarkar & Vijaya Vittala Institute of Technology & India \\
\hline Riyanarto Sarno & Institut Teknologi Sepuluh Nopember & Indonesia \\
\hline Mithileysh Sathiyanarayanan & City, University of London & $\begin{array}{l}\text { United Kingdom (Great } \\
\text { Britain) }\end{array}$ \\
\hline Soumya Sen & University of Calcutta, Kolkata & India \\
\hline Anindita Septiarini & Univeristas Mulawarman & Indonesia \\
\hline Amel Serrat & USTO MB & Algeria \\
\hline Arief Setyanto & Universitas AMIKOM Yogyakarta & Indonesia \\
\hline Iwan Setyawan & Satya Wacana Christian University & Indonesia \\
\hline Sfenrianto Sfenrianto & Bina Nusantara University & Indonesia \\
\hline Aditi Sharma & Quantum University, Roorkee, Uttarakhand & India \\
\hline Vesh Raj Sharma Banjade & Intel Corporation & USA \\
\hline China Sonagiri & Institute of Aeronautical Engineering & India \\
\hline lickho Song & Korea Advanced Institute of Science and Technology & Korea \\
\hline Yi-Jen Su & Shu-Te University & Taiwan \\
\hline Andi Sunyoto & Universitas AMIKOM Yogyakarta & Indonesia \\
\hline Nico Surantha & Bina Nusantara University & Indonesia \\
\hline Aries Susanto HT & UIN Syarif Hidayatullah Jakarta & Indonesia \\
\hline Takuji Tachibana & University of Fukui & Japan \\
\hline Srinivasulu Tadisetty & Kakatiya University College of Engineering and Technology & India \\
\hline Ivanna Timotius & Satya Wacana Christian University & Indonesia \\
\hline Ema Utami & Universitas Amikom Yogyakarta & Indonesia \\
\hline Julian Webber & Osaka University & Japan \\
\hline Leong Wen Chek & University of Malaya & Malaysia \\
\hline Ferry Wahyu Wibowo & Universitas Amikom Yogyakarta & Indonesia \\
\hline
\end{tabular}




\begin{tabular}{|c|c|c|}
\hline Teguh Wibowo & Gadjah Mada University & Indonesia \\
\hline Adhika Widyaparaga & Universitas Gadjah Mada & Indonesia \\
\hline Dedy Wijaya & Telkom University & Indonesia \\
\hline Warusia Yassin & Universiti Teknikal Malaysia Melaka & Malaysia \\
\hline Thaweesak Yingthawornsuk & King Mongkut's University of Technology Thonburi & Thailand \\
\hline Yuya Yokoyama & Kyoto Prefectural University & Japan \\
\hline Chau Yuen & Singapore University of Technology and Design & Singapore \\
\hline Go Yun II & Heriot-Watt University Malaysia & Malaysia \\
\hline Sri Zuliana & UIN Sunan Kalijaga & Indonesia \\
\hline Nur Zareen Zulkarnain & Universiti Teknikal Malaysia Melaka & Malaysia \\
\hline
\end{tabular}




\section{Additional Reviewers}

\begin{tabular}{|c|c|c|}
\hline Intan Ermahani A. Jalil & Universiti Teknikal Malaysia Melaka & Malaysia \\
\hline Mohd Helmy Abd Wahab & Universiti Tun Hussein Onn Malaysia & Malaysia \\
\hline Mohd Hakim Abdul Hamid & Faculty of Information Technology and Communication, UTeM & Malaysia \\
\hline David Agustriawan & Indonesia International Institute for Life Sciences (i3L) & Indonesia \\
\hline Mohd Khairul Ikhwan Bin Ahmad & Universiti Tun Hussein Onn Malaysia & Malaysia \\
\hline Anas Mohammad Ramadan AISobeh & Yarmouk University & Jordan \\
\hline Ahmad Ashari & Gadjah Mada University & Indonesia \\
\hline Aslina Baharum & Universiti Malaysia Sabah & Malaysia \\
\hline Aashish Bardekar & Sipna College of Engineering and Technology, Amravati & India \\
\hline Indra Budi & Faculty of Computer Science & Indonesia \\
\hline Maria Chiara Caschera & CNR & Italy \\
\hline Mu-Song Chen & Electrical Engineering, Da-Yeh University & Taiwan \\
\hline Wichian Chutimaskul & King Mongkut's University of Technology Thonburi & Thailand \\
\hline Domenico Ciuonzo & University of Naples Federico II, IT & Italy \\
\hline Gaia Codeluppi & University of Parma & Italy \\
\hline Ahmad Nurul Fajar & Bina Nusantara University & Indonesia \\
\hline Alireza Ghasempour & ICT Faculty & USA \\
\hline Razvan Andrei Gheorghiu & Politehnica University of Bucharest & Romania \\
\hline Hamdani Hamdani & Universitas Mulawarman & Indonesia \\
\hline Byeong-jun Han & Soongsil University & Korea \\
\hline Seng Hansun & Universitas Multimedia Nusantara & Indonesia \\
\hline K Haribabu & BITS Pilani & India \\
\hline Aslinda Hassan & Universiti Teknikal Malaysia Melaka & Malaysia \\
\hline Roberto Carlos Herrera Lara & National Polytechnic School & Ecuador \\
\hline Tonny Hidayat & Universitas AMIKOM Yogyakarta & Indonesia \\
\hline Danial Hooshyar & Korea University & Korea \\
\hline Ramkumar Jaganathan & VLB Janakiammal College of Arts and Science & India \\
\hline Biao Jiang & The City University of New York & USA \\
\hline Sokratis K. Katsikas & Norwegian University of Science and Technology & Norway \\
\hline Hasan Ali Ali Khattak & COMSATS University, Islamabad & Pakistan \\
\hline Suryadiputra Liawatimena & Bina Nusantara University & Indonesia \\
\hline Pavel Loskot & Swansea University & United Kingdom (Great Britain) \\
\hline Prita Dewi Mariyam & Universitas Indonesia & Indonesia \\
\hline Othman Mohd & Universiti Teknikal Malaysia Melaka & Malaysia \\
\hline Amrit Mukherjee & Jiangsu University & P.R. China \\
\hline Syibrah Naim & Universiti Sains Malaysia & Malaysia \\
\hline Ponrudee Netisopakul & King Mongkut's Institute of Technology Ladkrabang & Thailand \\
\hline Md Asri Ngadi & Universiti Teknologi Malaysia & Malaysia \\
\hline Ruzelita Ngadiran & Universiti Malaysia Perlis & Malaysia \\
\hline Hea Choon Ngo & Universiti Teknikal Malaysia Melaka & Malaysia \\
\hline Atsushi Nunome & Kyoto Institute of Technology & Japan \\
\hline Nitish Ojha & DIT University, Dehradun & India \\
\hline Henry Novianus Palit & Petra Christian University & Indonesia \\
\hline Jae-Hyun Park & Chung-Ang University & Korea \\
\hline Kiran Sree Pokkuluri & Shri Vishnu Engineering College for Women & India \\
\hline Gede Pramudya Ananta & Universiti Teknikal Malaysia Melaka & Malaysia \\
\hline Lesnanto Multa Putranto & UGM & Indonesia \\
\hline Yuansong Qiao & Athlone Institute of Technology & Ireland \\
\hline Basit Qureshi & University of Bradford & United Kingdom (Great Britain) \\
\hline Ali Rafiei & University of Technology Sydney & Australia \\
\hline Hemant Kumar Rath & Tata Consultancy Services & India \\
\hline Bagus Rintyarna & Sepuluh Nopember Institute of Technology & Indonesia \\
\hline Sayantam Sarkar & Vijaya Vittala Institute of Technology & India \\
\hline Riyanarto Sarno & Institut Teknologi Sepuluh Nopember & Indonesia \\
\hline Anindita Septiarini & Univeristas Mulawarman & Indonesia \\
\hline Amel Serrat & USTO MB & Algeria \\
\hline Iwan Setyawan & Satya Wacana Christian University & Indonesia \\
\hline Aditi Sharma & Quantum University, Roorkee, Uttarakhand & India \\
\hline
\end{tabular}




\begin{tabular}{|c|c|c|}
\hline China Venkateswarlu Sonagiri & Institute of Aeronautical Engineering & India \\
\hline lickho Song & Korea Advanced Institute of Science and Technology & Korea \\
\hline Yi-Jen Su & Shu-Te University & Taiwan \\
\hline Andi Sunyoto & Universitas AMIKOM Yogyakarta & Indonesia \\
\hline Nico Surantha & Bina Nusantara University & Indonesia \\
\hline Takuji Tachibana & University of Fukui & Japan \\
\hline Srinivasulu Tadisetty & Kakatiya University College of Engineering and Technology & India \\
\hline Ivanna Timotius & Satya Wacana Christian University & Indonesia \\
\hline Julian L Webber & Osaka University & Japan \\
\hline Leong Wen Chek & University of Malaya & Malaysia \\
\hline Ferry Wahyu Wibowo & Universitas Amikom Yogyakarta & Indonesia \\
\hline Teguh Wibowo & Gadjah Mada University & Indonesia \\
\hline Dedy Rahman Wijaya & Telkom University & Indonesia \\
\hline Yuhang Ye & Athlone Institute of Technology & Ireland \\
\hline Thaweesak Yingthawornsuk & King Mongkut's University of Technology Thonburi & Thailand \\
\hline Chau Yuen & Singapore University of Technology and Design & Singapore \\
\hline Go Yun II & Heriot-Watt University Malaysia & Malaysia \\
\hline Sri Utami Zuliana & UIN Sunan Kalijaga & Indonesia \\
\hline Nur Zareen Zulkarnain & Universiti Teknikal Malaysia Melaka & Malaysia \\
\hline
\end{tabular}




\section{International Conference on Information and Communications Technology (ICOIACT)}

1: Parallel Session 1-A

An Approach for High Bandwidth Wireless Communications with Arbitrary IQ Mismatch

Franz G Aletsee (Augsburg University of Applied Sciences, Germany), Reinhard Stolle (Hochschule Augsburg, Germany)

Identification and Prevention of Cyber Attack in Smart Grid Communication Network

Neeraj Singh (S V National Institute of Technology Surat, India), Vasundhara Mahajan (SVNIT, Surat, Gujarat \& IIT Roorkee, India)

Design of Vivaldi Antenna for UWB Respiration Radar

Tyas Oksi Praktika (Telkom University, Indonesia), Aloysius Adya Pramudita (Telkom University, Indonesia), Yuyu Wahyu (Indonesia Institute of Science LIPI, Indonesia)

ADS-B Microstrip Antenna Receiver Design for Cubesat with Slot

Essa Alkautsar Suteja (Telkom University, Indonesia), Agus D. Prasetyo (Telkom University, Indonesia), Bagas Satriyotomo (Telkom University, Indonesia), Desio Hasbin Dafiq (Telkom University, Indonesia), Edwar Edwar (Telkom University, Indonesia)

\section{Parallel Session 1-B}

Performance Evaluation of Active Queue Management in Fat Tree Architecture on Data Center Network

Lathifah Alfat (University of Indonesia, Indonesia)

Mobile-Based Geographic Information System For Culinary Tour Mapping In Indonesia

Erick Fernando (Bina Nusantara University, Indonesia), Muhamad Irsan (Universitas Islam Syekh Yusuf, Indonesia \& Universiti Kebangsaan Malaysia, Malaysia), Dina Fitria Murad, Dfm (Bina Nusantara University, Indonesia), Surjandy Surjandy (Bina Nusantara University, Indonesia), Djamaludin Djamaludin (Universitas Islam Syekh Yusuf (UNIS), Indonesia)

Learning Support System using Chatbot in "Kejar C Package" Homeschooling Program

Dina Fitria Murad, Dfm (Bina Nusantara University, Indonesia), Muhamad Irsan (Universitas Islam Syekh Yusuf, Indonesia \& Universiti Kebangsaan Malaysia, Malaysia), Erick Fernando (Bina Nusantara University, Indonesia), Silvia Murad (Universitas Islam Syekh Yusuf (UNIS), Indonesia), Michael Wijaya (Bina Nusantara University, Indonesia)

The Design of Two-Way Relationship Tourism Planning System with User Centered Design (UCD)

Yohandes Efindo (Universitas Gadjah Mada, Indonesia), Lukito Edi Nugroho (Universitas Gadjah Mada, Indonesia), Ridi Ferdiana (Universitas Gadjah Mada, Indonesia)

\section{Parallel Session 1-C}

An Image Steganography Algorithm using LSB Replacement through XOR Substitution

Touhid Bhuiyan (Daffodil International University, Bangladesh), Afjal H. Sarower (Daffodil International University, Bangladesh), Md. Rashed Karim (Daffodil International University, Bangladesh), Md. Maruf Hassan (Daffodil International University Dhaka Bangladesh \& Cyber Security Centre, DIU, Bangladesh)

A Novel Pseudo-Random Number Generator Algorithm based on Entropy Source Epoch Timestamp

Domingo Villanueva Origines, Jr (Technical Institute of the Philippines, Philippines)

A Modified Tiny Encryption Algorithm Using Key Rotation to Enhance Data Security for Internet of Things

Rey M. De Leon (Technological Institute of Philippines, Philippines), Ariel Sison (Emilio Aguinaldo College, Philippines), Ruji Medina (Technological Institute of Technology, Philippines)

$40 \mathrm{~Gb} / \mathrm{s}$ Balanced Parallel Scheme in Dispersion Compensating Fiber Performance for DWDM in the Long Haul Network

Brian Pamukti (Telkom University, Indonesia)

Parallel Session 1-D

Effectiveness Comparison of the AES and 3DES Cryptography Methods on Email Text Messages

Rini Indrayani (Universitas Amikom Yogyakarta, Indonesia), Subektiningsih Subektiningsih (Universitas Amikom Yogyakarta, Indonesia), Pramudhita Ferdiansyah (Universitas Amikom Yogyakarta, Indonesia), Dhimas Adi Satria (Universitas Amikom Yogyakarta, Indonesia)

Security Concern of Financial Technology for Online Transportation Passenger in Indonesia

Surjandy Surjandy (Bina Nusantara University, Indonesia), Erick Fernando (Bina Nusantara University, Indonesia), Firman Anindra (Universitas Nasional \& BINUS University, Indonesia), Meyliana Meyliana (Bina Nusantara University, Indonesia), Theresia Meidiana Santoso (Bina Nusantara University, Indonesia), Willy Widjaja (Bina Nusantara University, Indonesia), Anindya Wardhana (Bina Nusantara University, Indonesia)

Dual Protection on Message Transmission based on Chinese Remainder Theorem and Rivest Cipher 4

H. Kevin Cahyono (Dian Nuswantoro University, Indonesia), Christy Atika Sari (Dian Nuswantoro University, Indonesia), De Rosal Ignatius Moses Setiadi (Dian Nuswantoro University, Indonesia), Eko Hari Rachmawanto (Dian Nuswantoro University, Indonesia)

Collecting the Tourism Contextual Information data to support the tourism recommendation system

Rico Y Saputra (Universitas Gadjah Mada, Indonesia), Lukito Edi Nugroho (Universitas Gadjah Mada, Indonesia), Sri Suning

Kusumawardani (Universitas Gadjah Mada, Indonesia) 
Parallel Session 2-A

StegoCrypt Scheme using LSB-AES Base64

Fahmi Anwar (Dian Nuswantoro University, Indonesia), Eko Hari Rachmawanto (Dian Nuswantoro University, Indonesia), Christy Atika Sari (Dian Nuswantoro University, Indonesia), De Rosal Ignatius Moses Setiadi (Dian Nuswantoro University, Indonesia)

Segmentation of Plasmodium using Saturation Channel of HSV Color Space

Hanung Adi Nugroho (Universitas Gadjah Mada, Indonesia), Fathin Tantowi (Universitas Gadjah Mada, Indonesia), Raymond Anggara

(Universitas Gadjah Mada, Indonesia), TM Aldibra (Universitas Gadjah Mada, Indonesia), Rizki Nurfauzi (Gajah Mada Universeity,

Indonesia), Eka Frannita (Universitas Gadjah Mada, Indonesia), Alifia Prananda (Universitas Gadjah Mada, Indonesia)

Tuberculosis Detection in Chest X-Ray Images Using Optimized Gray Level Co-Occurrence Matrix Features

Imam Junaedi (Brawijaya University, Indonesia)

Rain Removal Using Guided Image Filtering For Surveillance Videos

Aditya Pratama Nusantara (Institut Teknologi Sepuluh Nopember, Indonesia), Budi Setiyono (Institut Teknologi Sepuluh Nopember, Indonesia), Dwi Ratna Sulistyaningrum (Sepuluh Nopember Institute of Technology, Indonesia), Izah Amalia (Institut Teknologi Sepuluh Nopember, Indonesia)

The Analysis Effect of Cluster Numbers On Fuzzy C-Means Algorithm for Blood Vessel Segmentation of Retinal Fundus Image Wiharto Wiharto (Universitas Sebelas Maret, Indonesia), Esti Suryani (University of Sebelas Maret, Indonesia)

Wood Classification with Transfer Learning Method and Bottleneck Features

Vajrayudha Ristiawanto (Telkom University, Indonesia), Budhi Irawan (Telkom University, Indonesia), Casi Setianingsih (Telkom University, Indonesia)

Prototype of Pornographic Image Detection using YCbCr and Color Space (RGB) Methods at Computer Vision

Kusrini Kusrini (AMIKOM Yogyakarta University, Indonesia), Hanif Fatta (Universitas AMIKOM Yogyakarta, Indonesia), Sofyan Pariyasto (University AMIKOM Yogyakarta, Indonesia), Wahyu Wijaya Widiyanto, www (University AMIKOM Yogyakarta, Indonesia)

Reduction of Inter-Cell Interference (ICI) by Fractional Frequency Reuse (FFR) in Orthogonal Frequency Division Multiple Access (OFDMA) Azlina Idris (Universiti Teknologi MARA, Malaysia)

\section{Parallel Session 2-B}

Temple Rock Damage Detection System in Digital Image at Borobudur Conservation Center

Ulfa Lutfiyana (Universitas Amikom Yogyakarta, Indonesia), Kusrini Kusrini (AMIKOM Yogyakarta University, Indonesia)

Classification of Palm Gesture Pattern by Using Statistical Features

Hendra Ari Winarno (Universitas Gadjah Mada \& Electrical and Information Technology Engineering, Indonesia), Indah Soesanti (Universitas Gadjah Mada, Indonesia), Hanung Adi Nugroho (Universitas Gadjah Mada, Indonesia)

A Programmable Artificial Neural Network Coprocessor for Handwritten Digit Recognition

Geranun Boonyuu (King Mongkut's Institute of Technology Ladkrabang, Thailand), Sumek Wisayataksin (King Mongkut's Institute of Technology Ladkrabang, Thailand)

Cirebon Mask Classification using Robust k- Nearest Neighbour

Felix Indra Kurniadi (Tanri Abeng University, Indonesia), Fendy Hendriyanto (Tanri Abeng University, Indonesia)

Analysis on Digital Elevation Model Data for 3D Modeling

Novandi Rezeki (Universitas AMIKOM Yogyakarta, Indonesia), Ema Utami (Universitas Amikom Yogyakarta, Indonesia), Irwan Oyong (Universitas AMIKOM Yogyakarta, Indonesia)

Adaptive Background Subtraction for Monitoring System

Afit Miranto (Universitas Lampung, Indonesia), Sri Ratna Sulistiyanti (University of Lampung, Indonesia), Arinto Setyawan (University of Lampung, Indonesia)

Segmentation and Recognition of Handwritten Lontara Characters Using Convolutional Neural Network

Asri Hidayat (Universitas Hasanuddin, Indonesia), Ingrid Nurtanio (Hasanuddin University, Indonesia), Zulkifli Tahir (Hasanuddin University, Indonesia)

A Developed Analysis Models for Industry 4.0 toward Smart Power Plant System Process

Harry Indrawan (PT PLN (Persero), Indonesia), Nur Cahyo (PT PLN (Persero), Indonesia), Arionmaro Simaremare (PT PLN (Persero), Indonesia), Siti Aisyah (PT PLN (Persero), Indonesia), P Paryanto (Universitas Diponegoro (UNDIP), Indonesia), Patrick Munyensanga (Universitas Diponegoro (UNDIP), Indonesia)

\section{Parallel Session 2-C}

An Improve Image Watermarking using Random Spread Technique and Discrete Cosine Transform Ajib Susanto (Universitas Dian Nuswantoro, Indonesia), De Rosal Ignatius Moses Setiadi (Dian Nuswantoro University, Indonesia), Eko Hari Rachmawanto (Dian Nuswantoro University, Indonesia), Ibnu Utomo Wahyu Mulyono (Dian Nuswantoro University, Indonesia), Christy Atika Sari (Dian Nuswantoro University, Indonesia)

Block-Based Arnold Chaotic Map for Image Encryption

Eko Hari Rachmawanto (Dian Nuswantoro University, Indonesia), De Rosal Ignatius Moses Setiadi (Dian Nuswantoro University, Indonesia), Christy Atika Sari (Dian Nuswantoro University, Indonesia), Heru Agus Santoso (Dian Nuswantoro University, Indonesia), Fauzi Adi Adi Rafrastara (Universitas Dian Nuswantoro, Indonesia), Edi Sugiarto (Dian Nuswantoro University, Indonesia) 
Image Steganography using Inverted LSB based on 2nd, 3rd and 4th LSB pattern

Fauzi Adi Adi Rafrastara (Universitas Dian Nuswantoro, Indonesia), Raka Prahasiwi (Dian Nuswantoro University, Indonesia), De Rosal Ignatius Moses Setiadi (Dian Nuswantoro University, Indonesia), Eko Hari Rachmawanto (Dian Nuswantoro University, Indonesia), Christy Atika Sari (Dian Nuswantoro University, Indonesia)

Copyright Embedding Analysis in Color Image Channel based on Non-Blind DCT Method

Fauzi Adi Adi Rafrastara (Universitas Dian Nuswantoro, Indonesia), Arvin Vega Hadinata (Dian Nuswantoro University, Indonesia), De Rosal Ignatius Moses Setiadi (Dian Nuswantoro University, Indonesia), Eko Hari Rachmawanto (Dian Nuswantoro University, Indonesia), Christy Atika Sari (Dian Nuswantoro University, Indonesia)

Strawberry Ripeness Classification System Based On Skin Tone Color using Multi-Class Support Vector Machine Indrabayu A (Hasanuddin University, Indonesia), Nurhikma Arifin (Hasanuddin University, Indonesia), Intan Sari Areni (Hasanuddin University, Indonesia)

Multimodal Interfaces: A Study on Speech-Hand Gesture Recognition

Jude Joseph Lamug Martinez (Bina Nusantara University \& Binus International, Indonesia), Sindy Dewanti (Binus University, Indonesia)

Recognition Pattern of Arca Siwa Prambanan Temple with Canny and Backpropagation Algorithm

Arif Fridasari (Universitas Amikom Yogyakarta, Indonesia), Ema Utami (Universitas Amikom Yogyakarta, Indonesia)

A Comparative Study MD5 and SHA1 Algorithms to Encrypt REST API Authentication on Mobile-based Application

De Rosal Ignatius Moses Setiadi (Dian Nuswantoro University, Indonesia), Afif Faishal Najib (Dian Nuswantoro University, Indonesia), Eko Hari Rachmawanto (Dian Nuswantoro University, Indonesia), Christy Atika Sari (Dian Nuswantoro University, Indonesia), Md Kamruzzaman Sarker (Wright State University, USA), Nova Rijati (Universitas Dian Nuswantoro, Indonesia)

\section{Parallel Session 2-D}

Optical Fiber Network Design in East Nusa Tenggara Based on Palapa Ring Project

Yohanes Galih Adhiyoga (Universitas Indonesia, Indonesia), Fetty Amelia (Universitas Indonesia, Indonesia), Dody Rachmat (Universitas Indonesia, Indonesia), B. Pratiknyo Adi Mahatmanto (Universitas Indonesia, Indonesia), Catur Apriono (Universitas Indonesia, Indonesia)

Data Distribution on the Goodness Behaviour System with Blackboard Based Architecture

Asa Hari Wibowo (Universitas Gadjah Mada, Indonesia), Lukito Edi Nugroho (Universitas Gadjah Mada, Indonesia), Selo Sulistyo (Gadjah Mada University, Indonesia)

Dual Band Antenna with Parasitic Patch for Satellite Applications

Harshal Nigam (Rajasthan Technical University, Kota \& SKIT M\&G, Engineering College, Jaipur, India), Monika Mathur (Rajasthan Technical University \& Swami Keshvanand Institute of Technology, Management and Gramothan, India), Mukesh Arora (SKIT Jaipur, India)

Optimization of Hyper Parameter Bandwidth on Naïve Bayes Kernel Density Estimation for the Breast Cancer Classification

Theopilus Bayu Sasongko (Universitas AMIKOM Yogyakarta, Indonesia), Oki Arifin (Politeknik Negeri Lampung, Indonesia), Hanif Fatta (Universitas AMIKOM Yogyakarta, Indonesia)

An Improved LBlock-s Key Schedule Algorithm

Arif Rahman Hakim (Sekolah Tinggi Sandi Negara, Indonesia), Zahra Zakia Nusron (Sekolah Tinggi Sandi Negara, Indonesia)

Self-Complementary Bow-tie Antenna Design for UWB Respiration System

Solihatul Jannah (Telkom University, Indonesia), Aloysius Adya Pramudita (Telkom University, Indonesia), Yuyu Wahyu (Indonesia Institute of Science LIPI, Indonesia)

A Control-Theoretical Perspective in Retail Telecommunication Industry Using Dynamic Simulation Model

Tiar Anindya Putri (Institut Teknologi Sepuluh Nopember, Indonesia), Riyanarto Sarno (Institut Teknologi Sepuluh Nopember, Indonesia),

Erma Suryani (Institut Teknologi Sepuluh Nopember, Indonesia)

\section{Parallel Session 3-A}

Hybrid NDP Proxy in OpenFlow Network

Fauzi Dwi Setiawan Sumadi (University of Muhammadiyah Malang, Indonesia), Ade Rega Susanto (University of Muhammadiyah Malang, Indonesia), Syaifuddin Syaifuddin (University of Muhammadiyah Malang, Indonesia), Didih Rizki Chandranegara (Universitas

Muhammadiyah Malang, Indonesia)

A Design of Digital Signature Mechanism in NDN-IP Gateway

Dian Abadi Arji (University of Indonesia, Indonesia), Fandhy Bayu Rukmana (University of Indonesia, Indonesia), Riri Fitri Sari (University of Indonesia, Indonesia)

An Introduction to a Dynamic Data Size Reduction Approach in Fog Servers

Mohammadreza Pourkiani (University of Rostock, Germany), Masoud Abedi (University of Rostock, Germany)

Improving the Quality of Service in WBSN Based Healthcare Applications by Using Fog Computing

Mohammadreza Pourkiani (University of Rostock, Germany), Masoud Abedi (University of Rostock, Germany), Mohammad Amin Tahavori

(Polytechnic University of Milan, Italy)

Blind Compressive Sensing for Cooperative Cognitive Radio with Semi-Orthognal RPC Matrix and I2-Minimization

Ahmed Ebian (Ain Shams University \& Telecom Egypt, Egypt) 
LTE-Advanced Network Planning Using Inter-band Non-Contiguous Carrier Aggregation Technology at Soreang-Pasir Koja Highway Yuyun Siti Rohmah (Telkom University, Indonesia), Sugondo Hadiyoso (Telkom University \& Institut Teknologi Bandung, Indonesia), Budi Prasetya (Institut Teknologi Bandung \& Telkom University, Indonesia)

Wall Effect compensation for Detection Improvement of Through the Wall Radar

Fauzan Nur A (Telkom University, Indonesia), Dharu Arseno (Telkom University, Indonesia), Aloysius Adya Pramudita (Telkom University, Indonesia)

Classification of Spice Types Using K-Nearest Neighbor Algorithm

Kaharuddin Kaharuddin (University of AMIKOM Yogyakarta, Indonesia), Kusrini Kusrini (AMIKOM Yogyakarta University, Indonesia), Vera Wati (University of AMIKOM Yogyakarta, Indonesia), Elvis Pawan (University Of AMIKOM Yogyakarta, Indonesia), Patmawati Hasan (University Of AMIKOM Yogyakarta, Indonesia)

Parallel Session 3-B

A Swing Routing Approach to Improve Performance of Shortest Geographical Routing Protocol for Wireless Sensor Networks Novi Trisman Hadi (Institut Teknologi Sepuluh Nopember Surabaya, Indonesia), Waskitho Wibisono (Institut Teknologi Sepuluh Nopember, Indonesia)

Experimental Measurement of Time Reversal-OFDM Technique for Underwater Acoustic Communication in the Presence of Gaussian Noise Yuning Widiarti (Institut Teknologi Sepuluh Nopember \& Politeknik Perkapalan Negeri Surabaya, Indonesia), Suwadi Suwadi (ITS, Indonesia), Wirawan Wirawan (Institut Teknologi Sepuluh Nopember, Indonesia)

Method to Uncover IP Spoofing Attack On Network Forensics Using NFAT And IP Correlation As Combined Approach Suryo Utomo (Institut Teknologi Bandung \& Badan Narkotika Nasional, Indonesia), Bayu Pramudiono (Badan Narkotika Nasional, Indonesia), Andika Muharam (Universitas Mercu Buana Jakarta, Indonesia)

Hjorth Descriptor as Feature Extraction for Classification of Familiarity in EEG Signal

Sugondo Hadiyoso (Telkom University \& Institut Teknologi Bandung, Indonesia), Inung Wijayanto (Telkom University \& Universitas Gadjah Mada, Indonesia), Hannisa Sanggarini (Telkom University, Indonesia)

Investigation of Human Emotion Pattern Based on EEG Signal Using Wavelet Families and Correlation Feature Selection Dwi Utari Surya (Brawijaya University, Indonesia)

A Study of Arousal Classification Based on EEG Signal and Support Vector Machine

Nur Arviah Sofyan (Telkom University, Indonesia), Inung Wijayanto (Telkom University \& Universitas Gadjah Mada, Indonesia), Sugondo Hadiyoso (Telkom University \& Institut Teknologi Bandung, Indonesia), Rita Purnamasari (Bandung Institute of Technology, Indonesia)

Analysis of Daubechies Wavelet and Neural Network for Audio Classification

Yulianto Mustaqim (Student of University \& AMIKOM Yogyakarta, Indonesia), Ema Utami (Universitas Amikom Yogyakarta, Indonesia), Suwanto Raharjo (Informatics Engineering of Institut Sains \& Teknologi AKPRIND Yogyakarta, Indonesia)

A simple real-time system for the detection of Myocardial Ischemia in the ST segment and T wave ECG signal

Prihatin Oktivasari (Politeknik Negeri Jakarta, Indonesia)

Parallel Session 3-C

Hoax Web Detection For News in Bahasa Using Support Vector Machine

Muhammad Abdillah Rahmat (Hasanuddin University, Indonesia), Indrabayu A (Hasanuddin University, Indonesia), Intan Sari Areni (Hasanuddin University, Indonesia)

Emotional Programmer's Behavior in Responding to Problems Using the Decision Tree

Agus Setiyono (STMIK Nusa Mandiri, Indonesia), Windu Gata (STMIK Nusa Mandiri, Indonesia)

Missing Values Estimation on Multivariate Dataset: Comparison of Three Type Method's Approach

Yoga Pristyanto (Universitas AMIKOM Yogyakarta, Indonesia), Irfan Pratama (Universitas Mercubuana Yogyakarta, Indonesia)

LTL Similarity and Classification using Fuzzy Rules for Evaluating Environment Sustainability Business Process Indicator

Lia Ninda Safitri (Institute Technology Sepuluh Nopember, Indonesia), Riyanarto Sarno (Institut Teknologi Sepuluh Nopember, Indonesia), Kelly Sungkono (Institut Teknologi Sepuluh Nopember, Indonesia)

Comparative Method of Moora and Copras Based on Weighting of the Best Worst Method in Supplier Selection at ABC Mining Companies in Indonesia

Ryco Setyono (Institut Teknologi Sepuluh Nopember, Indonesia), Riyanarto Sarno (Institut Teknologi Sepuluh Nopember, Indonesia)

Comparison Of MOORA and COPRAS Methods Based on Geographic Information System For Determining Potential Zone of Pasir Batu Mining

Adiba Ajrina (Institut Teknologi Sepuluh Nopember, Indonesia), Riyanarto Sarno (Institut Teknologi Sepuluh Nopember, Indonesia), R. V. Hari Ginardi (Institut Teknologi Sepuluh Nopember, Indonesia)

Application of Gravitational Search Algorithm to Detect Insulin Resistance in Intravenous Glucose Tolerance Test lim Abdul Mafahir (Bogor Agricultural University, Indonesia)

Predictive System Based Multi-layered Clustering Model and Least Absolute Shrinkage and Selection Operator (LASSO) Fevi Febianti (Institut Teknologi Bandung, Indonesia), Bambang Pharmasetiawan (Institut Teknologi Bandung, Indonesia), Kusprasapta Mutijarsa (Institut Teknologi Bandung, Indonesia) 
Designing Determining Teacher Engagement Based On The Indonesian Teacher Engagement Index Using Artificial Neural Network Sasmoko Sasmoko (Bina Nusantara University, Indonesia), Jurike Moniaga (Bina Nusantara University, Indonesia), Yasinta Indrianti (Research Interest Group in Educational Technology Bina Nusantara University, Indonesia), Yogi Udjaja (Bina Nusantara University, Indonesia), Christina Natasha (Bina Nusantara University, Indonesia)

Predicting the Potential Telemarketing Costumers using Data Mining Approach

Annisa Nurul Puteri (Hasanuddin University, Indonesia), Dewiani Dewiani (Universitas Hasanuddin, Indonesia), Zulkifli Tahir (Hasanuddin University, Indonesia)

Classification Talent of Employee Using C4.5, KNN, SVM, RBFN

Cecilia Stephanie (Institut Teknologi Sepuluh Nopember, Indonesia), Riyanarto Sarno (Institut Teknologi Sepuluh Nopember, Indonesia)

Software Quality Prediction Using Data Mining Techniques

Baydaa Mohammed Merzah (Al Nahrain University Iraq Baghdad, Iraq)

Optimal Sample Temperature of Electronic Nose For Detecting Beef And Pork Mixture

Sinarring Laga (Institut Teknologi Sepuluh Nopember, Indonesia), Riyanarto Sarno (Institut Teknologi Sepuluh Nopember, Indonesia)

Umrah Electronic Guide

Lolwah Alshabanat (General Authority of Zakat and Tax, Saudi Arabia), Areej AlHogail (King Saud University \& College of Computing and Information Sciences, Saudi Arabia), Nourah Abdulelah Almusharraf (Al-Imam Muhammad Ibn Saud Islamic University, Saudi Arabia), Arwa Ali Mahdi, Alkharis (Imam Muhammad ibn Saud Islamic University, Saudi Arabia), Bashair Almusharraf (Imam Muhammad Ibn Saud Islamic University, Saudi Arabia)

Quotation Extraction from Indonesian Online News

Achmad Choirudin Emcha (Universitas Gadjah Mada, Indonesia), Widy Widyawan (Gadjah Mada University, Indonesia), Teguh Bharata Adji (Universitas Gadjah Mada, Indonesia)

The Architecture of Tourism Recommendation System Based on Context-Awareness and Two-Way Relationship

Vivin Mahat Putri (Universitas Gadjah Mada, Indonesia), Lukito Edi Nugroho (Universitas Gadjah Mada, Indonesia), Adhistya Erna

Permanasari (Universitas Gadjah Mada, Indonesia)

Parallel Session 4-A

miRNA Based Gene Regulation of Bladder Cancer in A Specific Population of Caucasian Race and Different Sexes

Margareta Deidre Valeska (Indonesia International Institute for Life Sciences (i3L), Indonesia), David Agustriawan (Indonesia International Institute for Life Sciences (i3L), Indonesia \& AsiaUniversity, Taiwan)

Identification of microRNA Targeting Cancer Gene of Colorectal Carcinoma in Caucasian Population

Stefanus Bernard (Indonesia International Institute for Life Sciences, Indonesia), David Agustriawan (Indonesia International Institute for Life Sciences (i3L), Indonesia \& AsiaUniversity, Taiwan)

The Application of Extended Weighted Tree Similarity Algorithm for Similarity Searching

Akrilvalerat Deainert Wierfi (Universitas AMIKOM Yogyakarta, Indonesia), Ema Utami (Universitas Amikom Yogyakarta, Indonesia), Andi Sunyoto (Universitas AMIKOM Yogyakarta, Indonesia)

Chili Commodity Price Forecasting in Bandung Regency using the Adaptive Synthetic Sampling (ADASYN) and K-Nearest Neighbor (KNN) Algorithms

Hasmita S (Telkom University, Indonesia), Fhira Nhita (Telkom University, Indonesia), Deni Saepudin (Telkom University, Indonesia), Annisa Aditsania (Telkom University, Indonesia)

Time-Series Data Forecasting and Approximation with Smoothing Technique

Irfan Pratama (Universitas Mercubuana Yogyakarta, Indonesia), Putri Taqwa Prasetyaningrum (Mercu Buana University of Yogyakarta \& Faculty of Information Technology, Indonesia), Putry Wahyu Setyaningsih (Universitas Mercubuana Yogyakarta, Indonesia)

Business Trends Based on News Portal Websites for Analysis of Big Data Using K-Means Clustering

Wahyu Hidayat (University of Amikom Yogyakarta, Indonesia), Ainul Yaqin (Universitas Amikom Yogyakarta, Indonesia)

The Effect of Feature Selection on Classification Algorithms in Credit Approval

Yoga Pristyanto (Universitas AMIKOM Yogyakarta, Indonesia), Sumarni Adi (Universitas AMIKOM Yogyakarta, Indonesia), Andi Sunyoto (Universitas AMIKOM Yogyakarta, Indonesia)

Best Parameter Selection Of Rabin-Karp Algorithm In Detecting Document Similarity

Anggit Dwi Hartanto (Universitas Amikom Yogyakarta, Indonesia), Andy Syaputra (Universitas Amikom Yogyakarta, Indonesia), Yoga Pristyanto (Universitas AMIKOM Yogyakarta, Indonesia)

\section{Parallel Session 4-B}

Clustering of Javanese News in Krama Alus Level with Javanese Stemming

Denis Eka Cahyani (Universitas Sebelas Maret, Indonesia)

Sentiment Analysis on Grab User Reviews Using Support Vector Machine and Maximum Entropy

Annisa Uswatun Khasanah (Universitas Islam Indonesia, Indonesia), Bella Azis Dewanti Putri (Universitas Islam Indonesia, Indonesia), Abdullah Azzam (Industrial Engineering, Indonesia) 
The Impact of Using Domain Specific Features on Lexicon Based Sentiment Analysis on Indonesian App Review

Bayu Trisna Pratama (Universitas Amikom Yogyakarta, Indonesia), Ema Utami (Universitas Amikom Yogyakarta, Indonesia), Andi Sunyoto (Universitas AMIKOM Yogyakarta, Indonesia)

Social Media Mapping for Business Communication

Anggit Subekti (Universitas Gadjah Mada, Indonesia), Ridi Ferdiana (Universitas Gadjah Mada, Indonesia), Paulus Insap Santosa (Universitas Gadjah Mada, Indonesia)

Accuracy Measurement on Indonesian Non-formal Affixed Word Stemming With Levenhstein

Rahardyan Bisma Setya Putra (Universitas Amikom Yogyakarta, Indonesia), Ema Utami (Universitas Amikom Yogyakarta, Indonesia),

Suwanto Raharjo (Informatics Engineering of Institut Sains \& Teknologi AKPRIND Yogyakarta, Indonesia)

Literature Review of Automatic Text Summarization: Research Trend, Dataset and Method

Adhika Pramita Widyassari (Dian Nuswantoro University, Indonesia), Edy Noersasongko (Dian Nuswantoro University, Indonesia), Abdul

Syukur (Dian Nuswantoro University, Indonesia), Affandy Affandy (Universitas Dian Nuswantoro, Indonesia), Ahmad Zainul Fanani

(Universitas Dian Nuswantoro, Indonesia), Ruri Basuki (University of Dian Nuswantoro, Indonesia)

Real Time Face Expression Classification Using Convolutional Neural Network Algorithm

Vera Wati (University of AMIKOM Yogyakarta, Indonesia), Kusrini Kusrini (AMIKOM Yogyakarta University, Indonesia), Hanif Fatta

(Universitas AMIKOM Yogyakarta, Indonesia)

\section{Parallel Session 4-C}

Implementation of Rabin Karp Algorithm for Essay Writing Test System on Organization xyz

M Misbah Musthofa (Universitas Amikom Yogyakarta, Indonesia), Ainul Yaqin (Universitas Amikom Yogyakarta, Indonesia)

Implementation of Naive Bayes Algorithm for Spam Comments Classification on Instagram

Beta Priyoko (Universitas Amikom Yogyakarta, Indonesia), Ainul Yaqin (Universitas Amikom Yogyakarta, Indonesia)

Improving Random Forest Method to Detect Hatespeech and Offensive Word

Kristiawan Nugroho (Dian Nuswantoro University, Indonesia), Edy Noersasongko (Dian Nuswantoro University, Indonesia), Purwanto (Dian

Nuswantoro University, Indonesia), Muljono Muljono (Dian Nuswantoro University, Indonesia), Ahmad Zainul Fanani (Universitas Dian

Nuswantoro, Indonesia), Affandy Affandy (Universitas Dian Nuswantoro, Indonesia), Ruri Basuki (University of Dian Nuswantoro, Indonesia) ........ 514

Information Retrieval of Physical Force Using the TF-IDF

Dany Widiyatmoko (STMIK NUsa Mandiri Jakarta Indonesia, Indonesia), Agus Setiyono (STMIK Nusa Mandiri, Indonesia)

Mobile-Based Translation System for Cebuano Language with Object Detection for Travel Assistance using Neural Machine Translation Alonica R Villanueva (Technological Institute of the Philippines, Philippines), Reagan Balongcas (Technological Institute of the Philippines, Philippines), Aura Joy Aura Joy Baltazar (Technological Institute of the Philippines, Philippines), Bon Eric Rosete (Technological Institute of the Philippines, Philippines), Kim Omar Roxas (Technological Institute of the Philippines, Philippines), Johnathan Richard Barrios (Technological Institute of the Philippines, Philippines), Maria Cecilia Venal (Technological Institute of the Philippines, Philippines)

Text Normalization for Indonesian Abbreviated Word Using Crowdsourcing Method

Danny Sebastian (Duta Wacana Christian University, Indonesia), Kristian Nugraha (Duta Wacana Christian University, Indonesia)

Hate Speech Detection in Indonesian Language on Instagram Comment Section Using Maximum Entropy Classification Method

Elvira Erizal (Telkom University, Indonesia), Budhi Irawan (Telkom University, Indonesia), Casi Setianingsih (Telkom University, Indonesia)

Decision Support System Employee Recommendation using Fuzzy Sugeno Method as a Job Search Service

Kusnawi Kusnawi (AMIKOM University, Indonesia), Joang Ipmawati (University Of Nahdlatul Ulama Yogyakarta, Indonesia), Darma Kusumandaru Tri Prasetyo Utomo (Universitas Amikom Yogyakarta, Indonesia)

\section{Parallel Session 4-D}

Fuzzy K-Nearest Neighbor for Restaurants Business Sentiment Analysis on TripAdvisor

Baiq Billyan (Institut Teknologi Sepuluh Nopember, Indonesia), Riyanarto Sarno (Institut Teknologi Sepuluh Nopember, Indonesia), Kelly Sungkono (Institut Teknologi Sepuluh Nopember, Indonesia), Irene Tangkawarow (Institut Teknologi Sepuluh Nopember \& Universitas Negeri Manado, Indonesia)

Citation Detection on Scientific Journal Using Support Vector Machine

Raynaldi Amanullah (Amikom University, Indonesia), Ema Utami (Universitas Amikom Yogyakarta, Indonesia), Andi Sunyoto (Universitas AMIKOM Yogyakarta, Indonesia)

Discover the Indonesian Digital Workers in Online Gig Economy Platforms

A Labib Fardany Faisal (Universitas Indonesia, Indonesia), Yudho Sucahyo (University of Indonesia, Indonesia), Yova Ruldeviyani (Universitas Indonesia, Indonesia), Arfive Gandhi (Universitas Indonesia, Indonesia)

Apriori Algorithm Optimization using Temporary Table

Arif Dwi Laksito (Universitas Amikom Yogyakarta, Indonesia), Kusrini Kusrini (AMIKOM Yogyakarta University, Indonesia)

Performance Improvement of Recommender Hybrid Techniques Using GRU for Rating Calculation

Fernaldy Akbar Faudzan (Institut Teknologi Bandung, Indonesia), Bambang Pharmasetiawan (Institut Teknologi Bandung, Indonesia), Kusprasapta Mutijarsa (Institut Teknologi Bandung, Indonesia) 
A Conceptual Framework of Adaptive Mobile POI Recommendations

Emanuel Ristian Handoyo (Universitas Gadjah Mada, Indonesia), Selo Sulistyo (Gadjah Mada University, Indonesia), Paulus Insap Santosa (Universitas Gadjah Mada, Indonesia), Bimo Sunarfri Hantono (Universitas Gadjah Mada, Indonesia)

The Best Features Selection Method and Relevance Variable for Web Phishing Classification

Sumarni Adi (Universitas AMIKOM Yogyakarta, Indonesia), Yoga Pristyanto (Universitas AMIKOM Yogyakarta, Indonesia), Andi Sunyoto (Universitas AMIKOM Yogyakarta, Indonesia)

Implementation and Monitoring of Optimization of VLAN Networks with HTB and Multiple Hotspot Servers on University Scale Networks (Case Study: Immanuel Christian University)

Azriel Christian Nurcahyo (Universitas AMIKOM Yogyakarta \& Magister Teknik Informatika AMIKOM, Indonesia), Ema Utami (Universitas Amikom Yogyakarta, Indonesia), Suwanto Raharjo (Informatics Engineering of Institut Sains \& Teknologi AKPRIND Yogyakarta, Indonesia)

\section{Parallel Session 5-A}

Recommendations for Tourism Sites Using the Mamdani Fuzzy Logic Method and Floyd Warshall Algorithm (Case Study in Yogyakarta) Baltra Pramajuri (Universitas Atma Jaya Yogyakarta, Indonesia), Alfredo Gormantara (Universitas Atma Jaya Yogyakarta, Indonesia), Erni Widarti (Universitas Atma Jaya Yogyakarta, Indonesia), Albertus Joko Santoso (Universitas Atma Jaya Yogyakarta, Indonesia)

Convolutional Adversarial Neural Network (CANN) for Fault Diagnosis within a Power System

Ika Oktavianti (University of Sriwijaya, Indonesia), Steve Chan (Harvard University, USA)

Somnolence Detection System Utilizing Deep Neural Network

Alonica R Villanueva (Technological Institute of the Philippines, Philippines), Renzo Leru Benemerito (Technological Institute of the Philippines, Philippines), Mark Jetro Cabug-Os (Technological Institute of the Philippines, Philippines), Royce Chua (Technological Institute of the Philippines, Philippines), Cyrille Kristein Rebeca (Technological Institute of the Philippines, Philippines), Menchie Miranda (Technological Institute of the Philippines, Philippines)

A Comparison of Efficiency Improvement for Long Short-Term Memory Model Using Convolutional Operations and Convolutional Neural Network

Manop Phankokkruad (King Mongkut's Institute of Technology Ladkrabang, Thailand), Sirirat Wacharawichanant (Silpakorn University, Thailand)

An Application of Convolutional Neural Network-Long Short-Term Memory Model for Service Demand Forecasting

Manop Phankokkruad (King Mongkut's Institute of Technology Ladkrabang, Thailand), Sirirat Wacharawichanant (Silpakorn University, Thailand)

Query Optimization for Distributed Databases uses a Semi-join Based Approach (SBA) with the SDD-1 Algorithm

Ahmad Fikri Zulfikar (University of Pamulang \& Universiry of Bina Nusantara, Indonesia)

Performance Evaluation of Single Board Computer for Hadoop Distributed File System (HDFS)

Adnan Adnan (Universitas Hasanuddin, Indonesia), Zulkifli Tahir (Hasanuddin University, Indonesia), Muhammad Arfah Asis (Hasanuddin University, Indonesia)

Parallel Session 5-B

Ship Heading Control for Dubins Path Tracking and Collision Avoidance using Model Predictive Control

Dian Kusuma Rahma Putri (Institut Teknologi Sepuluh Nopember, Indonesia), Subchan Subchan (Institut Teknologi Sepuluh Nopember Indonesia), Dieky Adzkiya (Institut Teknologi Sepuluh Nopember, Indonesia), Tahiyatul Asfihani (Institut Teknologi Sepuluh Nopember, Indonesia)

An Investigation of RTOS-Based Sensor Data Management Performance for Tel-USat On Board Data Handling (OBDH) Subsystem Alif Rachman Harfian (Telkom University, Indonesia), Dharu Arseno (Telkom University, Indonesia), Edwar Edwar (Telkom University, Indonesia), Bagas Satriyotomo (Telkom University, Indonesia)

Robust Predictive Controller Application on Inventory Controlling with Imperfect Delivery Process

Sutrisno Sutrisno (Diponegoro University, Indonesia), Widowati Widowati (Diponegoro University, Indonesia)

Position Control of a Ship-Mounted Two-DoF Manipulator

Edwar Yazid (Research Center for Electrical Power and Mechatronics, Indonesian Institute of Sciences, Indonesia), Hendri Saputra (Indonesia Institute of Sciences, Indonesia), Midriem Mirdanies (Indonesian Institute of Sciences (LIPI), Indonesia), Rahmat Bubu (Indonesian Institute of Sciences, Indonesia)

Development and Control Segway by LQR adjustable Gain

Surachat Chantarachit (Rajamangala University of Technology Thanyaburi, Thailand)

Smoothed A-Star Algorithm for Nonholonomic Mobile Robot Path Planning

Syaiful Ardy Gunawan (Universitas Gadjah Mada, Indonesia), Gilang Nugraha Putu Pratama (Universitas Gadjah Mada \& Sekolah Tingg Teknologi Adisutjipto, Indonesia), Adha Imam Cahyadi (Universitas Gadjah Mada, Indonesia), Bondhan Winduratna (Universitas Gadjah Mada, Indonesia), Yohannes Chrysostomos Hendro Yuwono (Universitas Gadjah Mada, Indonesia), Oyas Wahyunggoro (UGM, Indonesia) ж........

Tunning of Fractional-Order PID Controller for Electro-Hydraulic Servo Valve System

Anggara Truna Negara (Gadjah Mada University, Indonesia)

Battery State of Charge Estimation Based on Coulomb counting Combined with Recursive Least Square and PI controller Agus Kustiman (Universitas Gadjah Mada, Indonesia), Bobby Dewangga (Universitas Gadjah Mada, Indonesia), Oyas Wahyunggoro (UGM, Indonesia), Adha Imam Cahyadi (Universitas Gadjah Mada, Indonesia) 
Parallel Session 5-C

Optimized Altitude Control for Quadrotor UAV in Virtual Environment

Ibnu Masngut (Universitas Gadjah Mada, Indonesia), Gilang Nugraha Putu Pratama (Universitas Gadjah Mada \& Sekolah Tinggi Teknologi Adisutjipto, Indonesia), Adha Imam Cahyadi (Universitas Gadjah Mada, Indonesia), Samiadji Herdjunanto (Universitas Gadjah Mada, Indonesia)

A New Industrial Robotics and Software Development Resolved the Position and the Speed Control

Dechrit Maneetham (Rajamangala University of Technology Thanyaburi \& TDS Thailand Company, Thailand)

Time and Cost Optimization in Feasibility Test of CCTV Project using CPM and PERT

Muhammad Bintang (Institut Teknologi Sepuluh Nopember, Indonesia), Kelly Sungkono (Institut Teknologi Sepuluh Nopember, Indonesia),

Riyanarto Sarno (Institut Teknologi Sepuluh Nopember, Indonesia)

Parallel Session 5-D

A Feasibility Study of Sliding Mode Controller Application for Hybrid Diesel-Electric Drive in Robot Defense System Ika Syamsiana (State Polytechnic of Malang, Indonesia), Fajar Kholid (State Polytechnic of Malang, Indonesia), R. Edy Purwanto (State Polytechnic of Malang, Indonesia)

Self Service System for Library Automation: Case Study at Telkom University Open Library

Nyoman Karna (Telkom University, Indonesia), Donny Pratama (Telkom University, Indonesia), Muhammad Ramzani (Telkom University, Indonesia)

Determining the Robust Counterpart of Flight Retiming Model

Khusnul Novianingsih (Universitas Pendidikan Indonesia, Indonesia)

Optimal Control of the Spread of Dengue Fever Using Dynamic Programming

Hartono Hartono (Sanata Dharma University, Indonesia)

Modeling and Simulation of Floating Droplet Using Multi-phase Lattice Boltzmann Method

Kumara Ari Yuana, Yun (Universitas Gadjah Mada \& Universitas Amikom Yogyakarta, Indonesia)

Balloon Trajectory: Monitoring, Prediction, and Analysis

Haryono Haryono (LAPAN, Indonesia).

Parallel Session 6-A

Terahertz Bow-tie Antenna-coupled Bolometer Impedance Matching by Transmission Line Matching Network

Arie Pangesti Aji (Universitas Indonesia, Indonesia), Eko Tjipto Rahardjo (Universitas Indonesia, Indonesia), Catur Apriono (Universitas Indonesia, Indonesia)

Rectangular Linear Array Microstrip Antenna Design for Terahertz Imaging

Intan Nurfitri (Universitas Indonesia, Indonesia), Catur Apriono (Universitas Indonesia, Indonesia)

Single-Phase DC-AC Inverter with Transformer and Transformerless and Low Power Dissipation Filter for Photovoltaic-Based Home-Scale Electric Power Systems

Ikhsan Hidayat (Hasanuddin University, Indonesia), Faizal Samman (University of Hasanuddin, Indonesia), Rhiza Sadjad (Hasanuddin University, Indonesia)

Performance Comparative study on DC-DC Boost Converters with Non-Isolated Configurations

Moh Afandy (Universitas Hasanudin, Indonesia), Faizal Samman (University of Hasanuddin, Indonesia), A Ejah Umraeni Salam (Electrical

Engineering \& Hassanuddin University, Indonesia)

Decomposition Wavelet Transform As Identification Of Outer Race Bearing Damage Through Stator Flow Analysis In Induction Motor Iradiratu Diah Prahmana karyatanti (Universitas Hang Tuah, Indonesia), Belly Yan Dewantara (Universitas Hang Tuah, Indonesia), Choirun Hida Hidayanto (Hang Tuah University, Indonesia)

comparison of Simple Battery Model and Thevenin Battery Model for SOC Estimation Based on OCV Method

Susanna Susanna (GadjahMada University \& Goverment Education, Indonesia), Oyas Wahyunggoro (UGM, Indonesia), Adha Imam

Cahyadi (Universitas Gadjah Mada, Indonesia), Bobby Dewangga (Universitas Gadjah Mada, Indonesia)

\section{Parallel Session 6-B}

Composite liquid insulators characteristics of palm and diala-b oil as transformer oil

Rizal Achmadsyah (Universitas Gadjah Mada, Indonesia), Sasongko Pramono Hadi (Gadjah Mada University, Indonesia), Sarjiya Sarjiya (Gadjah Mada University, Indonesia)

Effect of Distributed Photovoltaic Generation Installation on Voltage Profile: A Case Study of Rural Distribution System in Yogyakarta Indonesia

Anugrah Fitrah Gusnanda (Universitas Gadjah Mada, Indonesia), Sarjiya Sarjiya (Gadjah Mada University, Indonesia), Lesnanto Multa Putranto (UGM, Indonesia) 
FPGA-Based Electronic Pulse Generator for Single-Phase DC/AC Inverter

Muhamad Rusdi (Hasanuddin University, Indonesia), Faizal Samman (University of Hasanuddin, Indonesia), Rhiza Sadjad (Hasanuddin University, Indonesia)

Harmonic Mitigation Using Shunt Hybrid Power Filter in Departement of Electrical Engineering Universitas Negeri Malang Electrical Power System

Langlang Gumilar (Universitas Negeri Malang, Indonesia), Muhammad Afnan Habibi (Universitas Negeri Malang, Indonesia), Dwi Prihanto (Universitas Negeri Malang, Indonesia), Hendro Wicaksono (Karlsruhe Institute of Technology, unknown)

Analysis Performance Vertical Axis Wind Turbine Based on Pitch Angels to Output Power

Langlang Gumilar (Universitas Negeri Malang, Indonesia), Arya Kusumawardana (Universitas Negeri Malang, Indonesia), Dwi Prihanto (Universitas Negeri Malang, Indonesia), Hendro Wicaksono (Karlsruhe Institute of Technology, unknown)

Transformation of Thunderstorm Mechanisms into Computational Intelligence Applied to the Load Dispatch

AN Afandi (Universitas Negeri Malang, Indonesia \& Kumamoto University, Japan), Yunis Sulistyorini (IKIP Budi Utomo, Indonesia)

Optimal Sizing and Siting of PV-Based Distributed Generation for Losses Minimization of Distribution using Flower Pollination Algorithm Tegar Prasetyo (Universitas Gadjah Mada, Indonesia), Sarjiya Sarjiya (Gadjah Mada University, Indonesia), Lesnanto Multa Putranto (UGM, Indonesia)

Analysis of Load Fluctuation Effect on the Excitation Current of the Three-Phase Synchronous Generator at the Diesel Power Plant Ja'a Khusnul Huda (Universitas Negeri Malang, Indonesia), AN Afandi (Universitas Negeri Malang, Indonesia \& Kumamoto University, Japan

\section{Parallel Session 6-C}

Effect of Temperature Change of Liquid Isolator Based on Composite Diala B Oil and Palm Oil as Transformer Oil Dian Bagus Fachrurrozi (Universitas Gajah Mada, Indonesia), Sasongko Hadi (Universitas Gadjah Mada, Indonesia), Danang Wijaya (UGM, Indonesia)

Transmission Line Switching For Loss Reduction And Reliability Improvement

Atul Kumar Yadav (SVNIT SURAT, India), Vasundhara Mahajan (SVNIT, Surat, Gujarat \& IIT Roorkee, India)

Performance Comparison of Standard Boost Converter and Two-Phase Boost Converter

Beauty Anggraheny Ikawanty (Institut Teknologi Sepuluh November (ITS) \& Politeknik Negeri Malang (POLINEMA), Indonesia), Mochamad Ashari (Institut Teknologi Sepuluh Nopember (ITS) - Surabaya, Indonesia), Taufik Taufik (California Polytechnic State University, San Luis Obispo, USA), Dodi Garinto (Indonesian Power Electronics Center (IPEC) \& Politeknik Manufaktur Astra, Indonesia)

The Effect of Irradiance on Distribution Power System Stability in Large-Scale Grid-Connected Photovoltaic

Muammar Zainuddin (Universitas Ichsan Gorontalo, Indonesia), Frengki Surusa (Universitas Ichsan Gorontalo, Indonesia)

Optimal Design of Stator Slot Geometry for High-Speed Spindle Induction Motor Applications

Wawan Purwanto, WP (Universitas Negeri Padang \& UNP, Indonesia)

Dynamic Economic Dispatch for 150 kV Sulselbar power generation systems using Artificial Bee Colony Algorithm

Haripuddin Arsyad (Hasanuddin University, Indonesia), Ansar Suyuti (Hasanuddin of University, Indonesia), Sri Said (Hasanuddin University, Indonesia), Yusri Syam Akil (Hasanuddin University, Indonesia)

A Power Sharing Loop Control Method for Input-series Output-parallel Flyback-type Micro-Inverter Using Droop Method

Sandi Kurniawan (State Polytechnic of Malang, Indonesia), Ferdian Ronilaya (State Polytechnic of Malang, Indonesia), Mohammad Hidayat (State Polytechnic of Malang, Indonesia), Erfan Rohadi (Politeknik Negeri Malang, Indonesia), Indrazno Siradjuddin (State Polytechnic of Malang, Indonesia), Rachmat Sutjipto (State Polytechnic of Malang, Indonesia)

First Time User Experience Assessment on Web based Online Examination

Krisnawati Krisnawati (University of AMIKOM Yogyakarta, Indonesia), Mardhiya Hayaty (Universitas AMIKOM Yogyakarta, Indonesia), Bayu Setiaji (Universitas AMIKOM Yogyakarta, Indonesia), Arief Setyanto (Universitas AMIKOM Yogyakarta, Indonesia)

Parallel Session 6-D

Performance Evaluation of TEC1-12706 Thermoelectric Cooler Module at Low Temperature Experimentally

Elvira Salsabila (Telkom University, Indonesia), Tri Ayodha Ajiwiguna (Telkom University, Indonesia), Asep Suhendi (Telkom University, Indonesia)

State of Charge Estimation for Lithium Polymer Battery using Kalman Filter under Varying Internal Resistance

John Fisher Jefferson Pakpahan (Universitas Gadjah Mada, Indonesia), Bobby Dewangga (Universitas Gadjah Mada, Indonesia), Gilang Nugraha Putu Pratama (Universitas Gadjah Mada \& Sekolah Tinggi Teknologi Adisutjipto, Indonesia), Adha Imam Cahyadi (Universitas Gadjah Mada, Indonesia), Samiadji Herdjunanto (Universitas Gadjah Mada, Indonesia), Oyas Wahyunggoro (UGM, Indonesia)

Development of Flywheel Regenerative Capture System (FRCS) to Improve Electric Vehicle (EV) Energy Captured System

Agung Prijo Budijono (Institut Teknologi Sepuluh Nopember Surabaya (ITS), Indonesia)

Designing Knowledge Management System with Big Data for Hospital Inpatient Services (Case Study at Islamic Hospital XYZ Pekanbaru) Tommi Rahman Perdana (Bina Nusantara University, Indonesia), Siti Mujiatun (Bina Nusantara University, Indonesia), Sfenrianto Sfenrianto (Bina Nusantara University, Indonesia), Emil R. Kaburuan (IDEAS LAB, Indonesia) 
Redesign of E-Participation using User-Centered Design Approach for Improving User Experience

Wahid Hasim (Universitas Gajdah Mada, Indonesia), Sunu Wibirama (Universitas Gadjah Mada, Indonesia), Hanung Adi Nugroho (Universitas Gadjah Mada, Indonesia)

Designing Enterprise Architecture in Hospitals Group

Jordan Hakiki Sipahutar (Binus University, Indonesia), Faizal Asrul Pasaribu (Binus University, Indonesia), Bastian Paskal Situmorang (Binus University, Indonesia), Sfenrianto Sfenrianto (Bina Nusantara University, Indonesia), Emil R. Kaburuan (IDEAS LAB, Indonesia)

The Effect of Data Acquisition Techniques in Profiling Analysis Based On Twitter

Sumarni Adi (Universitas AMIKOM Yogyakarta, Indonesia), Anggit Dwi Hartanto (Universitas Amikom Yogyakarta, Indonesia), Ema Utami (Universitas Amikom Yogyakarta, Indonesia), Suwanto Raharjo (Informatics Engineering of Institut Sains \& Teknologi AKPRIND Yogyakarta, Indonesia), Irwan Oyong (Universitas AMIKOM Yogyakarta, Indonesia)

\section{Parallel Session 7-A}

Pregnancy Monitoring Mobile Application User Experience Assesment

Gunawan Wicahyono (Amikom University Yogyakarta, Indonesia), Arief Setyanto (Universitas AMIKOM Yogyakarta, Indonesia), Suwanto Raharjo (Informatics Engineering of Institut Sains \& Teknologi AKPRIND Yogyakarta, Indonesia), Arief Munandar (Universitas AMIKOM Yogyakarta, Indonesia)

Extending UTAUT2 to Explore Digital Wallet Adoption in Indonesia

Muhtarom Widodo (Institut Teknologi Sepuluh Nopember, Indonesia), Mohammad Irawan (Institut Teknologi Sepuluh Nopember, Indonesia), Rita Ambarwati (Universitas Muhammadiyah Sidoarjo, Indonesia)

Development of Educational Software for Electrical Engineering Subjects using MATLAB

Naim Nani Fadzlina (Universiti Teknologi Mara, Malaysia), Nur Syahida Mat Nusi (Universiti Teknologi MARA, Malaysia), Suzi Seroja Sarnin (University Technology MARA, Malaysia), Norsuzila Ya'acob (Universiti Teknologi Mara, Malaysia)

Readiness Indicators of Human Resources Aspects for MOOC Implementation

Ertanto Yohan Khrysdianto (Universitas Gadjah Mada, Indonesia), Sri Suning Kusumawardani (Universitas Gadjah Mada, Indonesia), Paulus Insap Santosa (Universitas Gadjah Mada, Indonesia)

Performance Analysis of Grid Interfaced Photovoltaic Systems for Reliable Agri- Microgrids using PVsyst

Totappa Shivlingappa Hasarmani (BVDUCOE \& BVCOEL Pune, India), Rajesh Holmukhe (Bharati Vidyapeeth University College of Engineering Pune India, India), Santosh Tamke (Sensycon Controls, India)

Parallel Session 7-B

Data Transmission in Machine to Machine Communication Protocols for Internet of Things Application: A Review

Thongdy Keophilavong (Indonesia, Indonesia)

New Home Energy Management Using loT In Smart Family

Gabriel Tamtama (Universitas Atma Jaya, Indonesia), Paulus Suryanto (Universitas Atma Jaya, Indonesia), Suyoto Suyoto (Universitas Atma Jaya Yogyakarta, Indonesia)

A Review: Design of Smart Home Electrical Management System Based on loT

Melky Radja (Universitas Atma Jaya Yogyakarta, Indonesia), Andi Wahju Rahardjo Emanuel (Universitas Atma Jaya Yogyakarta, Indonesia) .......... 910

Performance Analysis Spectrum Sensing using Eigenvalue-Moment-Ratio for Internet of Things Devices

Yasi Dani (Sekolah Tinggi Teknologi Kreatif Bina Nusantara Bandung, Indonesia), Mochammad Haldi Widianto (Sekolah Tinggi Teknologi Kreatif Bina Nusantara Bandung, Indonesia), Davy Ronald Hermanus (Sekolah Tinggi Teknologi Kreatif Bina Nusantara Bandung, Indonesia), Johan Muliadi Kerta (Sekolah Tinggi Teknologi Kreatif Bina Nusantara Bandung, Indonesia)

Selection of Scholarship Acceptance Using AHP and TOPSIS Methods

Patmawati Hasan (University Of AMIKOM Yogyakarta, Indonesia), Ema Utami (University of AMIKOM Yogyakarta \& University of AMIKOM Yogyakarta, Indonesia), Selviana Yunita (Universitas Of AMIKOM Yogyakarta, Indonesia), Elvis Pawan (University Of AMIKOM Yogyakarta, Indonesia), Kaharuddin Kaharuddin (University of AMIKOM Yogyakarta, Indonesia)

Parallel Session 7-C

Knowledge Management System Design for IT Troubleshooting (Case Study Biro TI BPK RI)

Mohammad Noversada Aprirashka (Universitas Gadjah Mada, Indonesia)

Master Data Management Maturity Model: A Case Study at Statistics Business Register in BPS-Statistics Indonesia Dewi Krismawati (Universitas Indonesia, Indonesia), Yova Ruldeviyani (Universitas Indonesia, Indonesia), Rinaldi Rusli (Universitas Indonesia, Indonesia)

Solving Multi-objective Vehicle Routing Problem Using Hyper-heuristic Method By Considering Balance of Route Distances

Sasmi Hidayatul Y T (Institut Teknologi Sepuluh Nopember, Indonesia), Arif Djunaidy (Institut Teknologi Sepuluh Nopember, Indonesia), Ahmad Muklason (Institut Teknologi Sepuluh Nopember, Indonesia)

Designing Cost Measurement System in A Small Scrum Based Software Company Using Activity Based Costing Model (Case Study: ABC Company)

Eko Agus Pramono (Institut Teknologi Sepuluh Nopember, Indonesia), Erma Suryani (Institut Teknologi Sepuluh Nopember, Indonesia) 
Measurement of Maturity Level Higher Education Governance Using Balanced Scorecard (BSC) and COBIT 4.1

Elvis Pawan (University Of AMIKOM Yogyakarta, Indonesia), Ema Utami (University of AMIKOM Yogyakarta \& University of AMIKOM Yogyakarta, Indonesia), Selviana Yunita (Universitas Of AMIKOM Yogyakarta, Indonesia), Patmawati Hasan (University Of AMIKOM

Yogyakarta, Indonesia), Kaharuddin Kaharuddin (University of AMIKOM Yogyakarta, Indonesia) 


\title{
A Review: Design of Smart Home Electrical Management System Based on IoT
}

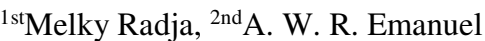 \\ Magister Teknik Informatika, University Atma Jaya Yogyakarta, Yogyakarta, Indonesia 55281 \\ ${ }^{1 \text { st }}$ melky.radjastudy@ gmail.com, ${ }^{2 n d}$ andi.emanuel@uajy.ac.id
}

\begin{abstract}
This paper will explain the benefits and optimal use of smart home energy management systems viewed in various aspects. The background of this paper is many problems in Indonesia regarding the consumption of electricity and the depletion of natural resources. Although the government has applied for an energy-saving program, the implementation program is still not optimal and has not been able to overcome the existing problems. In this paper, the idea of applying the SHEMS (Smart Home Electrical Management System) will be divided into three parts including IoT control, manual / automation control, and monitoring of real-time electrical energy. This research will also analyze the effectiveness of SHEMS in controlling energy use from several sources of journal literature. There are several points related to journal analysis including the effectiveness of the existing method, multi-objective scheduling, SHEMS design implementation and comparison of the results of After and before using SHEMS. From the results of the journal literature analysis, it is expected to help find the right SHEMS design method for each different case and suggests a framework for future systems.
\end{abstract}

Keywords: Smart Home, IoT, Sensor, Electrical management system, Control System, Real-time Monitoring System.

\section{INTRODUCTION}

Limited energy resources unable to overcome the demand of energy consumption which increases every time. Most of the problems faced in Indonesia is a continuous increase of consumers of electrical energy of year. Based on the statistical data the number of electricity use by consumers increases every year. The electrification ratio in Indonesia has reached $91.16 \%$ in 2016 and the used of oil energy for the country's power plant is 132.41 million BOE [1]. Based on this data the importance of energy savings by each community is an effective step which needs to be implemented.

IoT implementation on smart home energy management systems (SHEMS) has grown rapidly and answered many problems about energy savings in a smart home or smart city [2]. SHEMS is used to control various energy such us electrical energy, water, and gas. In the previous research, the implementation of SHEMS was carried out using various methods and different frameworks to obtain optimal energy savings results, such as the application of ZigBee sensors, automatic systems, manual controls and others, which can be seen in table 3. In this paper will be focus on efficiency electrical energy saving in smart home based IoT. Several functions of SHEMS that can be used is electrical energy monitoring [3], automatic energy controlling [4], manual controlling and electricity energy optimization.

This paper purpose to implement the most relevant SHEMS method through automatic and manual electrical energy control techniques using various sensors, real-time monitoring of electrical energy in smart home based on IoT, and its success rate.

\section{CURRENT STUDIES}

In the process of developing SHEMS, the selection of devices such as sensors and microcontrollers, architecture and proper implementation methods must be considered properly.

\section{A. Internet of Things}

The implementation of IoT architecture be required in the development of SHEMS. One of them is being able to guarantee security in the process of data communication management based on TCP / IP in SHEMS. In figure 1 is a six-layer architecture of IoT.

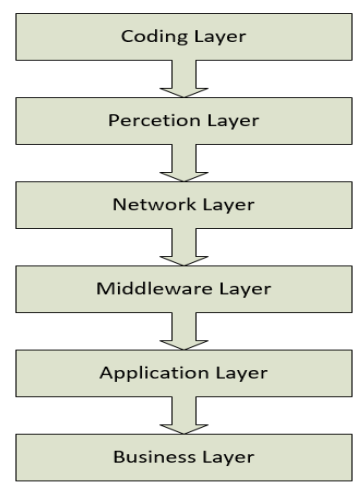

Figure 1: Six-Layer of IoT 
Figure 1 shows that IoT are composed of several layers Layers such as Perception Layer, Network Layer, Middleware Layer, Application Layer, and Business Layer for transmitted data through. IoT must have Radio Frequency Identification RFID [5], Wireless Sensor Network (WSN) [6] dan Claud computing IoT can be implemented in various sectors including, smart home, smart Trafic-System, Smart Grid and many more [5].

\section{A. The Effectiveness of SHEMS Devices controlling}

In this study, the SHEMS function is seen from how to control and monitor electrical energy through house device appliances that use electricity, including air conditioner (AC), lamp, television, and another device. From monitoring system, every home that applies SHEMS can monitor electrical energy consumption that used every day. Electrical data consumption can receive from anywhere using a smartphone. In terms of control, SHEMS can be controlled manually and automatically. Manually controlling can be done through Smartphone, where users can turn on / off the devices at home through an electric control panel application, and automatic controls will using sensor for each device. Example: use a motion sensor (PIR) on the lamp. The light will turn on automatically when there is movement captured by the sensor and will turn off automatically if there is no movement captured. Existing research on SHEMS, SHEMS based on IoT [7], Automatic Control [4], multi-sensor control [3], SHEMS from the consumer behavior [10], Realtime monitoring and Electrical Energy control [11] and real-time energy consumption information [12].

Based on the results of a literature review, success rate electrical energy consumption obtained in table 1.

Table 1. Literature Review of Success Rate Electrical Consumption

\begin{tabular}{|l|l|}
\hline \multicolumn{1}{|c|}{$\begin{array}{c}\text { Success Rate cost \& kWh Energy } \\
\text { consumption }\end{array}$} & Ref \\
\hline $18 \% \mathrm{kWh} /$ day & {$[13]$} \\
\hline $61.36 \% \mathrm{kWh} /$ Month & {$[4]$} \\
\hline $80 \% \mathrm{success}$ rate & {$[14]$} \\
\hline $10 \% \mathrm{kWh} /$ day & {$[10]$} \\
\hline
\end{tabular}

Table 1 shows the success rate of implementing SHEMS. The success rate is based on the amount of energy consumption before and after the implementation of SHEMS. From the results of the percentage amount of electrical energy consumption in table 1 success depends on the type of framework used.

\section{B. Sensor Appliances}

A sensor is the part of IoT Perception Layer which can be used to changing information from connected devices to analog signals. In implementation of SHEMS there are several studies that combine more than one sensor for smart home energy management system such like, temperature and humidity sensor for controlling air conditioner [15], temperature and humidity sensor using for different region [16], temperature and humidity sensor for controlling fan [17], PIR sensor for control lights by detecting the heat from occupants moving [4], Light Sensor [17], Meters Sensor [18], Sound sensor [19], Infrared sensor [14].

\section{Smart Home Appliances}

To build an electricity management system the main thing to consider is what kind of smart home appliances should be implemented using IoT. the determination of the type of smart home equipment results in a large amount of electrical energy consumption that can be optimized SHEMS through IoT.

Table 2. Several Types of Devices

\begin{tabular}{|c|c|}
\hline Smart Home Appliances & $\begin{array}{c}\text { Journals } \\
\text { References }\end{array}$ \\
\hline Lamp & $\begin{array}{l}{[20],[13], \quad[10],} \\
{[15],[7],[16]}\end{array}$ \\
\hline Fan & {$[15],[16]$} \\
\hline $\mathrm{AC}$ & $\begin{array}{l}{[20],[13],[15],} \\
{[7],[16]}\end{array}$ \\
\hline Phone Charger & {$[13],[16]$} \\
\hline Refrigerator & {$[13],[16]$} \\
\hline Boiler & {$[13],[16]$} \\
\hline TV & {$[13],[14],[16]$} \\
\hline $\begin{array}{l}\text { Cameras for security, } \\
\text { Electric Clothes Dryer, } \\
\text { Coffee Machine, } \\
\text { Microwave, Blender, LCD } \\
\text { Monitor, Well Pump (1/3-1 } \\
\text { HP), Clothes Dryer, } \\
\text { Washing machine, Vacum } \\
\text { cleaner }\end{array}$ & {$[16]$} \\
\hline
\end{tabular}

Table 2 shows about several types of home appliances that are monitored and controlled using smartphone and various sensor via SHEMS. 
The application of sensors to each home appliances must be in accordance with their respective functions. Sensor fault determination will result in the waste of energy. Example: if the motion sensor is placed on the fan, the fan will keep ON whenever the sensor reads the movement. For, the most appropriate sensor used is a temperature sensor. The fan will be $\mathrm{ON}$ only if the temperature of room is too hot.

\section{Existing Framework}

Determining the right framework is an important thing that can affect the success rate of reducing the amount of energy consumption. The framework used should be low in cost and must be in accordance with the conditions in which it is implemented.

Table 3: Existing Framework

\begin{tabular}{|c|c|}
\hline Framework & Ref. \\
\hline $\begin{array}{l}\text { Real-time monitoring temperature and } \\
\text { humidity monitoring system based on } \\
\text { IoT with Multi-sensor Implementation. }\end{array}$ & {$[3]$} \\
\hline $\begin{array}{l}\text { IoT-based real-time monitoring system } \\
\text { with multi-sensor implementation to } \\
\text { compare the accuracy of data received } \\
\text { from each sensor. }\end{array}$ & [21] \\
\hline $\begin{array}{l}\text { The recommended system for an energy } \\
\text { control on a smart home based on } \\
\text { occupants activities or habits. }\end{array}$ & {$[10]$} \\
\hline $\begin{array}{l}\text { Automat system with multi-sensor and } \\
\text { monitoring system uses local home } \\
\text { network. }\end{array}$ & {$[4]$} \\
\hline $\begin{array}{l}\text { Manually controlling system via a } \\
\text { smartphone using Infra Red (IR). }\end{array}$ & [14] \\
\hline $\begin{array}{l}\text { Two manual control systems using Wifi } \\
\text { for indoor controllers and using GSM } \\
\text { modules for control from outside the } \\
\text { home. }\end{array}$ & [13] \\
\hline $\begin{array}{l}\text { Automat control system and monitor } \\
\text { energy based on the amount of costs to be } \\
\text { paid. }\end{array}$ & [15] \\
\hline $\begin{array}{l}\text { The application of the ZigBee sensor, the } \\
\text { system can be monitored and controlled } \\
\text { manually via a Smartphone. }\end{array}$ & [11] \\
\hline $\begin{array}{l}\text { distributed optimization algorithm for } \\
\text { scheduling the energy consumption of } \\
\text { multiple smart homes using a smart } \\
\text { meter. }\end{array}$ & [22] \\
\hline $\begin{array}{l}\text { Home appliances controlling using } \\
\text { ZigBee and IR remote control. }\end{array}$ & [23] \\
\hline $\begin{array}{l}\text { Electrical consumption optimization, } \\
\text { residential appliances control, smart } \\
\text { plugs, and Conservation Voltage } \\
\text { Reduction (CVR) }\end{array}$ & [24] \\
\hline $\begin{array}{l}\text { Electrical appliances using wireless } \\
\text { smart socket. }\end{array}$ & [25] \\
\hline $\begin{array}{l}\text { Manual and Automatic control light } \\
\text { based on IoT. }\end{array}$ & [7] \\
\hline
\end{tabular}

Table 3 describes some of the frameworks used in existing studies. Determining the right framework can support the effectiveness of SHEMS. The determination of the framework must be adjusted to the problems faced, the application environment, and costs.

\section{E. Novelty of SHEMS}

The application ideas for Smart home energy management system will be different with the existing framework. Design Framework concerning SHEMS system will combine multi-sensor (temperature and humidity sensor, PIR sensor, light sensor, meters sensor, and sound sensor), multicontrolling for every single device (automatic and manual controlling), real-time energy monitoring using smartphone, Home prototype design and SHEMS implementation is adapted to the application environment.

\section{SHEMS IMPLEMENTATION METHODS}

In smart home energy management system based on IoT there are a few main points that must be considered there are components used, different architectural designs for each building, and what type of control will be implemented to the system.

\section{A. IoT Smart Home System Component}

Smart home electrical management system is a system built from supporting components such as microcontrollers, sensor relays, and routers. Each component has its own role and function to support the energy saving process in smart homes. The following are some of the components used to build SHEMS:

1. Microcontroller will be used is a Master Controller S3C6410 [3] as the data control center of each sensor and connected device.

2. Sensors: Temperature and humidity sensor for air conditioner controlling [15] and fan controlling [17], PIR sensor for lamp controlling [4], Light Sensor for outdoor lamp controlling [17], Meters Sensor [18], and Sound sensor for lamp controlling [19]. Every sensor will get the information data from object and then converted to electrical signals. The function of sensors is as an automatic control system connected with microcontroller as a central data processing.

3. Relay plays an important role as an electrical switch that can be run with a logic function and will be connected to any home electronic device with 220V voltage [17] [18].

4. Wifi Router : in this journal Wifi Home is used to connect each device to the internet. 


\section{A. Method for Design Smart Home energy system}

Based on a review of several existing methods in this paper, the Idea for SHEMS design method was given. The idea of developing the system includes determining the installation of SHEMS, determining the system controlling and monitoring.

1. System design is built based on the extent and function of the room for determining the use of suitable sensors, electrical installations used to determine the position of the micro-controller board and the design of the SHEMS architecture and determine the control function of each device.

2. The device control design can be run automatically by using sensors. System will receive sensor data reading and determine the condition value. When the system receives analog data sent by the sensor according to the value of the specified condition, the device will automatically ON / OFF.

3. The manual control system will use smartphone as a control panel.

4. The system is able to monitor electricity used $(\mathrm{kWh} /$ day) online via a smartphone from anywhere.

5. The controlling system can be selected according to the user's wishes.

\section{IMPLEMENTATION AND CONSTRUCTION}

This Section will discuss the idea of the implementation and construction of the system design. Different building types affect the effectiveness of energy savings. Determination of sensor installation and IoT network installation on the smart home should be based on the area of the room, the function of the room, the installation of the electricity network used, and the range of sensors and the ability of the sensor to capture data.

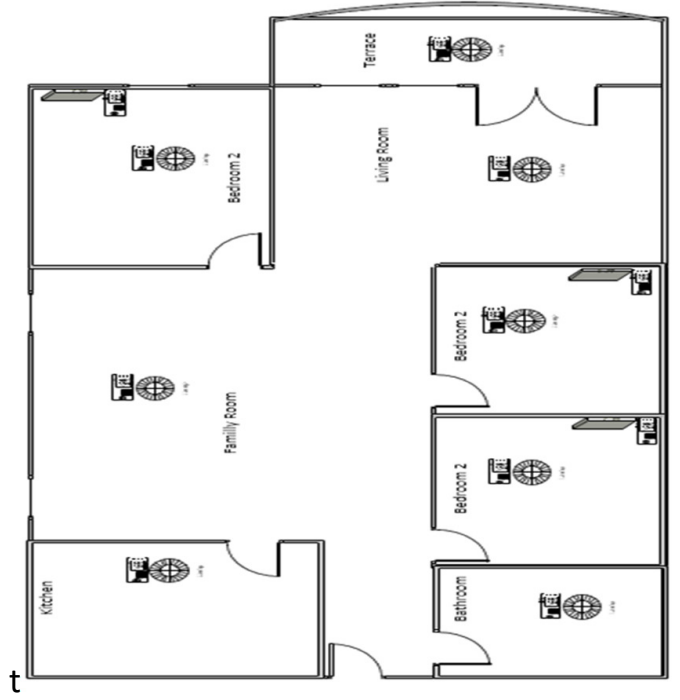

Figure 2 : home sketch
Figure 2 is a display of an example of a house plan design where the position and placement of each sensor is adjusted to the function of the device used in each room. Sensor placement is considered from several aspects including the function of the room, activity, volume of the room, the function of electrical equipment, and the position of the placement of sensors must be able to reach the entire room.

In addition to building types, another thing that must be considered is the type of system control. Every equipment in the house must be able to control the use of electrical energy. How to control electrical energy from each equipment must be in accordance with the function of the equipment itself. The control systems used include automatic control of equipment, manual control and combine the control system. In Figure 3 shows the process of the automatic controlling system.

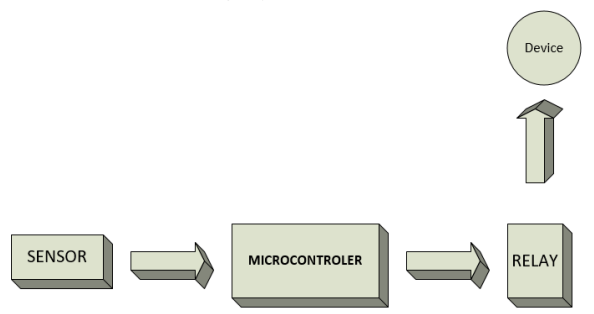

Figure 3: Automatic Control System

The sensor will send data to the microcontroller and the microcontroller will analyze the data received by the sensor according to the conditions specified in the analog data value limit. The microcontroller will forward the relay command in the form of a logic function to turn on or turn off the device.

Other system control functions are controlled manually, without involving sensor to control system. In figure 4 is the manual working principle.

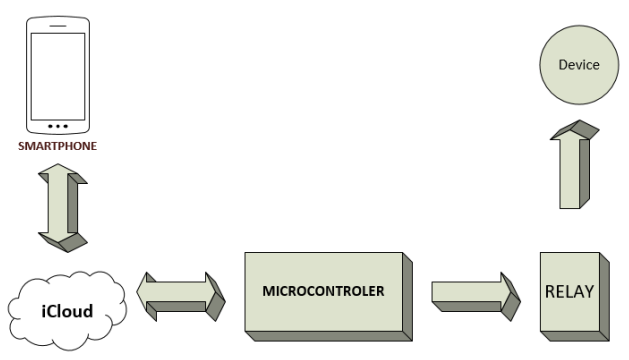

Figure 4: Manual Control System

In manual control systems, each connected device can be controlled via the control panel application installed on smartphones using the internet. Orders sent via a smartphone will be 
received by the microcontroller and immediately relayed to turn on or turn off the device.

In addition to the manual and automatic control functions, the monitoring function is also needed to monitor the amount of electricity consumption. Figure 5 shows an overview of the electrical energy monitoring system.

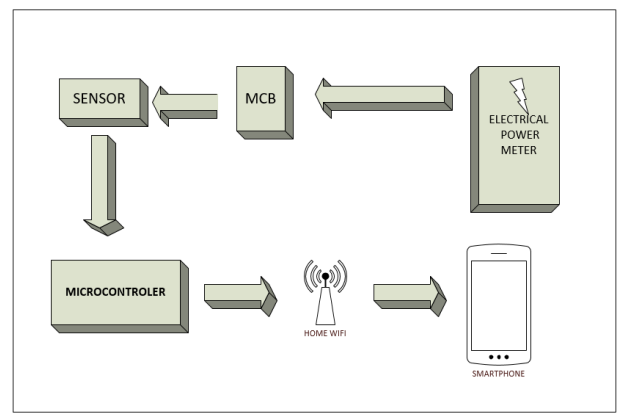

Figure 5: electrical energy monitoring system

The position of meters sensor placement is adjusted to the electrical network installation. The system electrical monitoring does not monitor each device, but the overall use of electrical energy in the house.

In this paper, one additional control function is a dual control function. Figure 6 shows an electrical multi controlling system architecture.

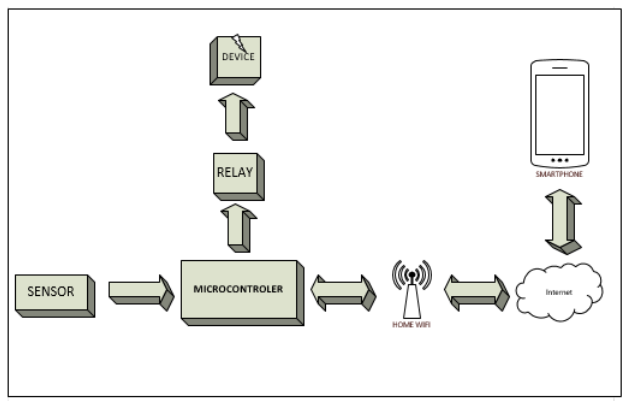

Figure 6: Multi-Controlling system

The system is able to control one device that is the same as the two control methods, namely manual and automatic control. The application of two control functions on one device uses the logic OR function on the relay.

\section{CONCLUSION}

This paper presents a description of the main components of SHEMS as well as various frameworks that are used to get the effectiveness of SHEMS. The strategy and implementation process are seen based on the type of device used, type of controlling, communication system used, and optimization techniques of using sensors. In this paper also given an idea about the method of implementing sensors based on the function of the room and the movement activity of the occupants of the house. The purpose of this paper is to find the appropriate framework and provide ideas about the incorporation of frameworks from various literature studies in the implementation of low-cost, efficient SHEMS and are able to answer the problem of energy waste that occurs in Indonesia. Another goal is to encourage people to understand how to use less energy at certain times and how to install electrical equipment to save electricity costs through SHEMS.

For further development, the system is expected to be able to build a smart home network where each house in the city is integrated with each other in a network, the system is also expected to be able to deliver data on the use of each household equipment not only to the homeowner but also to the state electricity company to be able to monitor the savings of electricity resources of each consumer.

\section{REFERENCES}

[1] Indonesia, "2010 Handbook of Energy \& Economic Statistics of Indonesia," pp. 1-50, 2009.

[2] A. Bhati, M. Hansen, and C. M. Chan, "Energy conservation through smart homes in a smart city: A lesson for Singapore households," Energy Policy, vol. 104, no. May, pp. 230-239, 2017.

[3] L. Guo, G. Wang, and X. Yu, "Design for indoor environment monitoring system based on embedded system and multi-sensor data fusion algorithm," Int. J. Smart Home, vol. 10, no. 1, pp. 31-40, 2016.

[4] I. I. Attia and H. Ashour, "Energy saving through smart home," Online J. Power Energy Eng., vol. 2Attia, I., no. 3, pp. 223-227, 2011.

[5] M. U. Farooq and M. Waseem, "A Review on Internet of Things ( IoT )," Int. J. Comput. Appl. (0975 8887), vol. 113 , no. 1 , pp. 1-7, 2015.

[6] S. S. Rajpoot and A. Khandelwal, "Home Energy Control System Using Wireless Smart Socket and IoT," Electron. Comput. Eng., 2018.

[7] T. Education, W. Shyr, L. Zeng, C. Lin, C. Lin, and W. Hsieh, "Application of an Energy Management System via the Internet of Things on a University Campus," vol. 14, no. 5, pp. 1759-1766, 2018.

[8] J. Kim, "HEMS (home energy management system) base on the IoT smart home," Contemp. Eng. Sci., vol. 9, no. 1, pp. 21-28, 2016.

[9] P. R. Joshi and M. S. Prof, "IOT Based Smart Power Management System Using WSN," Int. Res. J. Eng. Technol., vol. 4, no. 6, pp. 783-786, 2017.

[10] H. Wache, H. F. Witschel, H. F. Zanatta, M Rodriguez, and M. Zehnder, "Energy saving in smart homes based on consumer behavior: A case study," First IEEE Int. Smart Cities Conf., 2015.

[11] W. H. Kim, S. Lee, and J. Hwang, "Real-time energy monitoring and controlling system based on ZigBee sensor networks," Procedia Comput. Sci., vol. 5, pp. 794-797, 2011.

[12] M. Peruzzini et al., "Smart Home Information Management System for Energy-Efficient Networks To cite this version : HAL Id : hal-01463233 Smart Home Information Management System for," 2017.

[13] B. Mubdir, A. Al-Hindawi, and N. Hadi, "Design of Smart Home Energy Management System for Saving Energy," Eur. Sci. J., vol. 12, no. 33, 2016.

[14] J. Dvorak, O. Berger, and O. Krejcar, "Universal Central Control of Home Appliances as an Expanding Element of the Smart Home Concepts - Case Study on Low Cost Smart Solution," Comput. Inf. Syst. Ind. 
Manag. Cisim 2014, vol. 8838, pp. 479-488, 2014 R. Panna, R. Thesrumluk, and C. Chantrapornchai, "Development of Energy Saving Smart Home Prototype," Int. J. Smart Home, vol. 7, no. 1, pp. 4766, 2013.

[16] K. A. Fakeeh, "An IoT based Smart Power Mangement System for Technical University," Int. J. Comput. Appl., vol. 149, no. 1, pp. 975-8887, 2016.

[17] B. Bharmal, A. Shahapurkar, and A. Aswalkar, "Automatic Home Lighting solutions using Human Detection, Sunlight Intensity and Room

Temperature," Int. Res. J. Eng. Technol., vol. 4, no. 6, pp. 691-696, 2017.

[18] M. J. Mnati, A. Van den Bossche, and R. F. Chisab, "A smart voltage and current monitoring system for three phase inverters using an android smartphone application," Sensors (Switzerland), vol. 17, no. 4, pp. 1-16, 2017.

[19] C. Zyskowski and M. C. de Oliveira, "An Analog Audio Sensor Board for Microcontrollers," J. Audio Eng. Sco, no. November 2016, pp. 1-5, 2017.

[20] I. I. I. Attia, H. Ashour, E. Inji, I. I. I. Attia, and P. Hamdy, "Energy Saving Through Smart Home," Online J. Power Energy Eng., vol. 2, no. 3, pp. 223227, 2011.

[21] S. Lee, J. Park, I. Eng, and I. Eng, "Design of IoT based fire-watching and Atmospheric Environment Monitoring Systems Applied with Compound Sensors and Image Processing," vol. 10, no. 12, pp. 155-168, 2016.

[22] I. Y. Joo and D. H. Choi, "Distributed Optimization Framework for Energy Management of Multiple Smart Homes with Distributed Energy Resources," IEEE Access, vol. 5, pp. 15551-15560, 2017.

[23] J. Han, C. S. Choi, and I. Lee, "More efficient home energy management system based on ZigBee communication and infrared remote controls," IEEE Trans. Consum. Electron., vol. 57, no. 1, pp. 85-89, 2011.

[24] A. Parsa, T. A. Najafabadi, and F. R. Salmasi, "Implementation of smart optimal and automatic control of electrical home appliances (IoT)," IEEE Proc. 2017 Smart Grid Conf. SGC 2017, vol. 2018Janua, pp. 1-6, 2018.

[25] K. L. Tsai, F. Y. Leu, and I. You, "Residence Energy Control System Based on Wireless Smart Socket and IoT," IEEE Access, vol. 4, pp. 2885-2894, 2016. 
